\title{
Adaptive Identification and Control of Hysteresis in Smart Materials
}

\author{
Xiaobo Tan, Member, IEEE, and John S. Baras, Fellow, IEEE
}

\begin{abstract}
Hysteresis hinders the effective use of smart materials in sensors and actuators. This paper addresses recursive identification and adaptive inverse control of hysteresis in smart material actuators, where hysteresis is modeled by a Preisach operator with a piecewise uniform density function. Two classes of identification schemes are proposed and compared, one based on the hysteresis output, the other based on the time-difference of the output. Conditions for parameter convergence are presented in terms of the input to the Preisach operator. An adaptive inverse control scheme is developed by updating the Preisach operator (and thus its inverse) with the output-based identification method. The asymptotic tracking property of this scheme is established, and for periodic reference trajectories, the parameter convergence behavior is characterized. Practical issues in the implementation of the adaptive identification and inverse control methods are also investigated. Simulation and experimental results based on a magnetostrictive actuator are provided to illustrate the proposed approach.
\end{abstract}

Index Terms-Adaptive control, hysteresis, identification, inversion, Preisach operator, smart materials.

\section{INTRODUCTION}

$\mathbf{S}$ MART materials, e.g., magnetostrictives, piezoelectrics, and shape memory alloys (SMAs), show the coupling of mechanical properties with applied electromagnetic/thermal fields and hence have built-in sensing/actuation capabilities. However, strong hysteresis existing in these materials hinders their effective use in sensors and actuators [1]. The hysteresis behavior often varies slowly with time, which makes the hysteresis control problem even more challenging. To address this issue, recursive identification and adaptive control algorithms are developed in this paper based on a special class of Preisach hysteresis operators.

Hysteresis models can be roughly classified into physicsbased models and phenomenological models. An example of a physics-based model is the Jiles-Atherton model of ferromagnetic hysteresis [2], where hysteresis is considered to arise from pinning of domain walls on defect sites. The most popular phenomenological hysteresis model used for smart materials has been the Preisach operator [3]-[10], where the

Manuscript received November 3, 2004; revised March 10, 2005. Recommended by Associate Editor E. Bai. This work was supported by the Army Research Office under the ODDR\&E MURI97 Program Grant DAAG55-97-10114 to the Center for Dynamics and Control of Smart Structures (through Harvard University, Cambridge, MA) and by the Lockheed Martin Chair Endowment Funds.

$X$. Tan is with the Department of Electrical and Computer Engineering, Michigan State University, East Lansing, MI 48824 USA (e-mail: xbtan@msu.edu).

J. S. Baras is with the Institute for Systems Research and the Department of Electrical and Computer Engineering, University of Maryland, College Park, MD 20742 USA (e-mail: baras@isr.umd.edu).

Digital Object Identifier 10.1109/TAC.2005.849215 hysteresis is modeled as a (weighted) aggregate effect of all possible delayed relay elements, which are parameterized by a pair of threshold variables [11]. A similar type of operator, called Krasnosel'skii-Pokrovskii (KP) operator [12], [13], has also been used for modeling hysteresis in smart materials [14], [15]. The difference between the Preisach operator and the $\mathrm{KP}$ operator is that delayed relay elements in the latter have finite slopes. Another phenomenological hysteresis model is the Prandtl-Ishlinskii (PI) operator [16]. The PI model is a superposition of elementary play or stop operators, which are parameterized by a single threshold variable [13].

A fundamental approach in coping with hysteresis is inverse compensation, where one aims to cancel out the hysteresis effect by constructing a right inverse of the hysteresis model (see, e.g., [4], [8]-[10], [15], and [17]-[19]). It has been widely observed that hysteretic behaviors of smart materials vary with time, temperature and some other ambient conditions. Hence the performance of open-loop inverse compensation based on a fixed model is susceptible to model uncertainties. To combat this problem, a robust control framework was proposed by combining inverse compensation with $l_{1}$ control theory [20]. An alternative approach is adaptive inverse control [16], [17], [21], where the inverse model is updated adaptively. Tao and Kokotović developed an adaptive inverse control scheme for a class of hysteresis models with piecewise linear characteristics cascaded with known or unknown linear dynamics [17]. Adaptive identification and inverse control were studied for a discretized KP operator and applied to an SMA actuator by Webb et al. [21]. Kuhnen and Janocha examined an adaptive inverse scheme for piezoelectric actuators, where the PI hysteresis operator was used [16].

What distinguish this paper from [16], [17], and [21] are: 1) the Preisach operator is used to model the hysteresis nonlinearity; 2) the persistent excitation (PE) conditions for parameter convergence are presented in terms of the input to the hysteresis operator; and 3) the asymptotic tracking property of the adaptive inverse control algorithm is proved, and for periodic reference trajectories, the parameter convergence behavior is characterized.

For the Preisach operator, the model "parameter" is the Preisach density function. A classical method to identify the density function is to twice differentiate the Everett surface obtained with first order reversal inputs [11]. Researchers adopted functions of specific forms to fit the Everett surface based on the least-squares method [4], [7] or fuzzy/neural network approximators [10]. Another identification method is to devise the input carefully and derive the Preisach weighting masses (on a discretization grid) directly from the output measurements (solving for $n$ unknowns from $n$ equations) [22]. This scheme is very sensitive to measurement noises as one can easily see. 
A third approach is to identify the Preisach weighting masses or the weights for basis functions based on the least-squares method [8], [14], [15], [23]. The aforementioned identification methods were all used offline, nonrecursively. In this paper, hysteresis is modeled by a Preisach operator with a density function that is constant within each cell of the discretization grid, and the density values are identified recursively. Two recursive identification algorithms are proposed and compared, one based on the hysteresis output and the other based on the time-difference of the output.

A necessary condition and a sufficient condition for parameter convergence are given in terms of the input to the Preisach operator. In contrast to persistent excitation conditions for identification of linear systems, which are centered around the number of frequency components of the input signal, the conditions here involve the reversal behavior of the input. For ease of presentation, the analysis is done primarily for a Preisach operator with discrete weighting masses (obtained by assuming the density function within each cell is concentrated as a mass at the cell center). The extension to the case of a piecewise constant density is then briefly outlined.

An adaptive inverse control scheme is developed by updating the Preisach model (and thus its inverse) with the output-based identification algorithm. It is shown in this paper that, with the adaptive inverse scheme, the tracking error approaches zero asymptotically. Furthermore, for periodic reference trajectories, the parameter convergence behavior under this scheme can be characterized if the reference trajectory visits the positive or negative saturation value. On the other hand, it is shown that adaptive inverse control using the difference-based algorithm for parameter update cannot achieve asymptotic tracking. These results are illustrated and verified through experiments of controlling a magnetostrictive actuator.

Identification and inversion of a Preisach operator with piecewise uniform density, were also involved in the authors' prior work [20]. However, there the identification was done offline without looking into the identifiability issue, and the inversion algorithm was developed for models with known densities. Although the inversion scheme in [20] will be used in this paper, establishing the convergence of the adaptive control algorithm is new. Preliminary versions of some results reported in this paper were presented at ACC'04 and NOLCOS'04 [24], [25].

The remainder of this paper is organized as follows. For the convenience of the reader, the Preisach operator is briefly reviewed in Section II. Recursive identification of the hysteresis model is studied in Section III, and adaptive inverse control is discussed in Section IV. Finally, some concluding remarks are provided in Section $\mathrm{V}$.

\section{Preisach Operator AND A Discretization Scheme}

\section{A. The Preisach Operator}

Detailed treatment on the Preisach operator can be found in [11], [13], and [26]. For a pair of thresholds $(\beta, \alpha)$ with $\beta \leq \alpha$, consider a simple hysteretic element $\hat{\gamma}_{\beta, \alpha}[\cdot, \cdot]$, as illustrated in Fig. 1. Let $C([0, T])$ denote the space of continuous functions on $[0, T]$. For $u \in C([0, T])$ (the space of continuous functions on

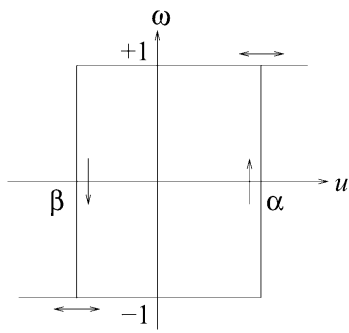

Fig. 1. Elementary Preisach hysteron $\hat{\gamma}_{\beta, \alpha}[\cdot, \cdot]$.

$[0, T])$ and an initial configuration $\zeta \in\{-1,1\}, \omega=\hat{\gamma}_{\beta, \alpha}[u, \zeta]$ is defined as, for $t \in[0, T]$

$$
\omega(t) \triangleq \begin{cases}-1, & \text { if } u(t)<\beta \\ 1, & \text { if } u(t)>\alpha \\ \omega\left(t^{-}\right), & \text {if } \beta \leq u(t) \leq \alpha\end{cases}
$$

where $\omega\left(0^{-}\right)=\zeta$ and $t^{-} \triangleq \lim _{\epsilon>0, \epsilon \rightarrow 0} t-\epsilon$.

This operator is sometimes referred to as an elementary Preisach hysteron (called hysteron hereafter in this paper), since it is a building block of the Preisach operator. Define

$$
\mathcal{P}_{0} \triangleq\left\{(\beta, \alpha) \in \mathbb{R}^{2}: \beta \leq \alpha\right\}
$$

$\mathcal{P}_{0}$ is called the Preisach plane, and each $(\beta, \alpha) \in \mathcal{P}_{0}$ is identified with the hysteron $\hat{\gamma}_{\beta, \alpha}$. For $u \in C([0, T])$ and a Borel measurable initial configuration $\zeta_{0}$ of all hysterons, $\zeta_{0}: \mathcal{P}_{0} \rightarrow$ $\{-1,1\}$, the output of the Preisach operator $\Gamma$ is defined as [13]

$$
y(t)=\Gamma\left[u, \zeta_{0}\right](t)=\int_{\mathcal{P}_{0}} \mu(\beta, \alpha) \hat{\gamma}_{\beta, \alpha}\left[u, \zeta_{0}(\beta, \alpha)\right](t) d \beta d \alpha
$$

where the weighting function $\mu$ is often referred to as the Preisach function [11] or the density function [26]. Throughout this paper it is assumed that $\mu \geq 0$. Furthermore, to simplify the discussion, assume that $\mu$ has a compact support, i.e., $\mu(\beta, \alpha)=0$ if $\beta<\beta_{0}$ or $\alpha>\alpha_{0}$ for some $\beta_{0}, \alpha_{0}$. In this case, it suffices to consider a finite triangular area in the Preisach plane $\mathcal{P} \triangleq\left\{(\beta, \alpha) \in \mathcal{P}_{0} \mid \beta \geq \beta_{0}, \alpha \leq \alpha_{0}\right\}$ (see Fig. 2(a)).

The memory effect of the Preisach operator can be captured by the memory curves in $\mathcal{P}$. At time $t, \mathcal{P}$ can be divided into two regions

$$
\begin{aligned}
& \mathcal{P}_{+}(t) \triangleq\left\{(\beta, \alpha) \in \mathcal{P} \mid \text { output of } \hat{\gamma}_{\beta, \alpha} \text { at } t \text { is }+1\right\} \\
& \mathcal{P}_{-}(t) \triangleq\left\{(\beta, \alpha) \in \mathcal{P} \mid \text { output of } \hat{\gamma}_{\beta, \alpha} \text { at } t \text { is }-1\right\}
\end{aligned}
$$

Now, assume that at some initial time $t_{0}$, the input $u\left(t_{0}\right)=$ $u_{0}<\beta_{0}$. Then, the output of every hysteron is -1 . Therefore, $\mathcal{P}_{-}\left(t_{0}\right)=\mathcal{P}, \mathcal{P}_{+}\left(t_{0}\right)=\emptyset$ and this corresponds to the "negative saturation" [Fig. 2(b)]. Next, assume that the input is monotonically increased to some maximum value at $t_{1}$ with $u\left(t_{1}\right)=u_{1}$. The output of $\hat{\gamma}_{\beta, \alpha}$ is switched to +1 as the input $u(t)$ increases past $\alpha$. Thus, at time $t_{1}$, the boundary between $\mathcal{P}_{-}\left(t_{1}\right)$ and $\mathcal{P}_{+}\left(t_{1}\right)$ is the horizontal line $\alpha=u_{1}$ [Fig. 2(c)]. Next assume that the input starts to decrease monotonically until it stops at $t_{2}$ with $u\left(t_{2}\right)=u_{2}$. It is easy to see that the output of $\hat{\gamma}_{\beta, \alpha}$ becomes -1 as $u(t)$ sweeps past $\beta$, and correspondingly, a vertical line segment $\beta=u_{2}$ is generated as part of the 


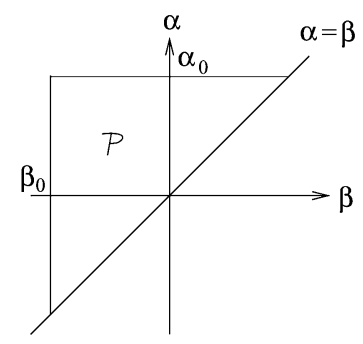

(a)

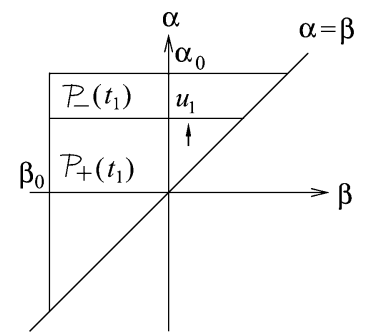

(c)

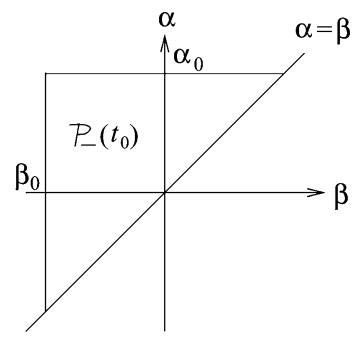

(b)

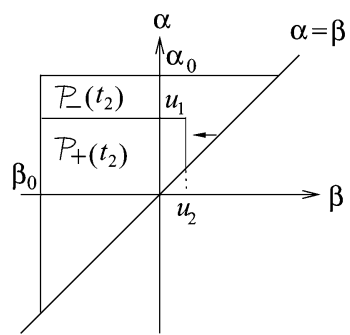

(d)
Fig. 2. Illustration of memory curves on the Preisach plane.

boundary [Fig. 2(d)]. Further input reversals generate additional horizontal or vertical boundary segments.

From the prvious illustration, each of $\mathcal{P}_{-}$and $\mathcal{P}_{+}$is a connected set [11], and the output of $\Gamma$ is determined by the boundary between $\mathcal{P}_{-}$and $\mathcal{P}_{+}$. This boundary iscalled the memorycurve sinceitcharacterizes the states of all hysterons. The memory curve has a staircase structure and its intersection with the line $\alpha=\beta$ gives the current input value. The set of allmemory curves is denoted $\Psi$. The memory curve $\psi_{0}$ at $t=0$ is called the initial memory curve and it represents the initial condition of the Preisach operator. Hereafter, the initial memory curve will be put as the second argument of the Preisach operator.

Rate-independence is one of the fundamental properties of the Preisach operator.

Theorem 2.1 (Rate-Independence [13]): If $\phi:[0, T] \rightarrow$ $[0, T]$ is an increasing continuous function satisfying $\phi(0)=0$ and $\phi(T)=T$, then for $u \in C([0, T]), \Gamma\left[u \circ \phi, \psi_{0}\right](t)=$ $\Gamma\left[u, \psi_{0}\right](\phi(t)), \forall t \in[0, T]$, where "o" denotes composition of functions.

\section{B. Discretization of the Preisach Operator}

In practice, the Preisach operator needs to be discretized in one way or another during the identification process. A natural way to approximate a Preisach operator is to assume that within each cell of the discretized Preisach plane, the Preisach density function is constant. This approximation has nice convergence (to the true Preisach operator) properties under mild assumptions [22].

Let $\left[u_{\min }, u_{\max }\right]$ be the practical input range to the hysteresis operator, which is often a strict subset of $\left[\alpha_{0}, \beta_{0}\right]$. For the hysteretic behavior one can focus on the triangle bounded by $u_{\min }$ and $u_{\max }$ in the Preisach plane, since the contribution to the output from hysterons outside this triangle is constant [8]. Discretize $\left[u_{\min }, u_{\max }\right]$ uniformly into $L+1$ levels (called discretization of level $L$ in this paper), where the discrete input levels $u_{i}, 1 \leq i \leq L+1$, are defined as

$$
u_{i}=u_{\min }+(i-1) \Delta_{u}
$$

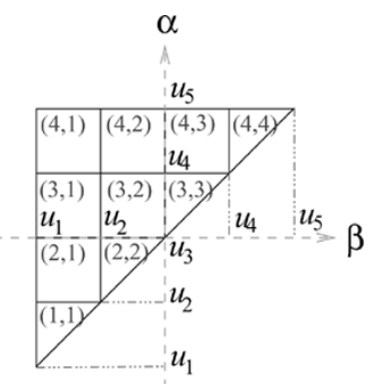

(a)

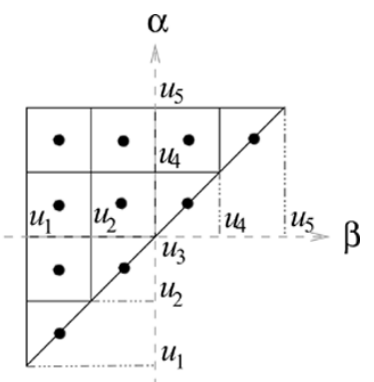

(b)
Fig. 3. Illustration of the discretization scheme $(L=4)$. (a) Labeling of the disretization cells. (b) Weighting masses sitting at the centers of cells.

with $\Delta_{u}=\left(u_{\max }-u_{\min } / L\right)$. The cells in the discretization grid are labeled, as illustrated in Fig. 3(a) for the case of $L=4$.

In this paper, by a piecewise uniform density function, we will specifically mean one that is constant within each discretization cell. Note that a Preisach operator with such a density function is still an infinite-dimensional operator. If one assumes that the Preisach density function inside each cell is concentrated at the center as a weighting mass [Fig. 3(b)], the corresponding Preisach operator becomes a weighted combination of a finite number of hysterons, which is a hysteretic, finite automaton [27].

\section{RECURSIVE IDENTIFICATION OF HYSTERESIS}

\section{A. Recursive Identification Schemes}

A Preisach operator with discrete weighting masses can only take input in a finite set $\left\{u_{i}\right\}_{i=1}^{L+1}$ and its set of memory curves is finite. These properties make it easier to analyze than a Preisach operator with piecewise uniform density. On the other hand, these two types of operators bear much similarity and essential results for one can be easily translated into those for the other. Hence, recursive identification of Preisach weighting masses is first studied, and then the extension needed for identifying the piecewise uniform density is briefly pointed out.

In the interest of digital control, the discrete-time setting is considered in this paper. To avoid ambiguity one should understand that the input to the Preisach operator is monotonically changed from $u[n-1]$ to $u[n]$. Two classes of identification algorithms are examined, one based on the hysteresis output, and the other based on the time difference of the output (called difference-based hereafter).

Output-Based Identification: The output $y[n]$ of the discretized Preisach operator [corresponding to the case illustrated in Fig. 3(b)] at time instant $n$ can be expressed as

$$
y[n]=\sum_{i=1}^{L} \sum_{j=1}^{i} \bar{W}_{i j}[n] \bar{\nu}_{i j}^{*}
$$

where $\bar{W}_{i j}[n]$ denotes the state $(1$ or -1$)$ of the hysteron in cell $(i, j)$ at time $n$, and $\bar{\nu}_{i j}^{*}$ denotes the hysteron's Preisach weighting mass. Stacking $\bar{W}_{i, j}[n]$ and $\bar{\nu}_{i, j}^{*}$ into two vectors, $W[n]=\left[W_{1}[n] \ldots W_{K}[n]\right]^{T}$ and $\nu^{*}=\left[\nu_{1}^{*} \ldots \nu_{K}^{*}\right]^{T}$, where $K=L(L+1) / 2$ is the number of cells, one rewrites (2) as

$$
y[n]=\sum_{k=1}^{K} W_{k}[n] \nu_{k}^{*}=W[n]^{T} \nu^{*} .
$$


Let $\hat{\nu}[n]=\left[\hat{\nu}_{1}[n] \ldots \hat{\nu}_{K}[n]\right]^{T}$ be the estimate of $\nu^{*}$ at time $n$, and let

$$
\hat{y}[n]=\sum_{k=1}^{K} W_{k}[n] \hat{\nu}_{k}[n]=W[n]^{T} \hat{\nu}[n]
$$

be the predicted output based on $\hat{\nu}[n]$. The gradient algorithm [28] to update the estimate is

$$
\hat{\nu}[n+1]=\hat{\nu}[n]-\gamma \frac{(\hat{y}[n]-y[n]) W[n]}{W[n]^{T} W[n]}
$$

where $0<\gamma<2$ is the adaptation constant. To ensure that the weighting masses are nonnegative, we let $\hat{\nu}_{k}[n+1]=0$ if the $k$ th component of the right hand side of (5) is negative. Since this parameter projection step brings the parameter estimate closer to the true values, it does not invalidate convergence results obtained without consideration of this step [28]. Hence, it will not be explicitly used during convergence analysis in this paper (in particular, in the proof of Theorem 4.1).

Difference-Based Identification: An alternative way to identify $\nu^{*}$ is using the time difference $z[n]$ of the output $y[n]$, where

$$
z[n] \triangleq y[n]-y[n-1]=(W[n]-W[n-1])^{T} \nu^{*} .
$$

Let $\hat{y}\left[n^{-}\right]$and $\hat{y}[n-1]$ be the output predictions at time $n$ and $n-1$ based on $\hat{\nu}[n-1]$, respectively, i.e.,

$$
\begin{gathered}
\hat{y}\left[n^{-}\right] \triangleq W[n]^{T} \hat{\nu}[n-1] \\
\hat{y}[n-1] \triangleq W[n-1]^{T} \hat{\nu}[n-1] .
\end{gathered}
$$

Define

$$
\hat{z}[n] \triangleq \hat{y}\left[n^{-}\right]-\hat{y}[n-1]=(W[n]-W[n-1])^{T} \hat{\nu}[n-1] .
$$

Let $V[n]$ be the time difference of hysteron states, $V[n] \triangleq$ $W[n]-W[n-1]$. Then we can obtain the following identification scheme based on $z[n]$ :

$$
\hat{\nu}[n+1]= \begin{cases}\hat{\nu}[n]-\gamma \frac{(\hat{z}[n]-z[n]) V[n]}{V[n]^{T} V[n]}, & \text { if } V[n] \neq 0 \\ \hat{\nu}[n], & \text { if } V[n]=0 .\end{cases}
$$

As in the output-based scheme, an additional parameter projection step will be applied if any component of $\hat{\nu}[n+1]$ is negative.

Having discussed the methods for recursive identification of weighting masses for a Preisach operator, we now explain how to change the previous algorithms for identification of the (piecewise uniform) Preisach density. In this case, the input is no longer limited to a finite set of values; instead it can take any value in $\left[u_{\min }, u_{\max }\right]$. The output $y[n]$ can still be expressed as (2) or (3), but with different interpretations for $\bar{W}_{i, j}[n]$ and $\bar{\nu}_{i, j}^{*}$. $\bar{W}_{i, j}[n]$ no longer represents the state (1 or -1$)$ of the hysteron at the center of the cell $(i, j)$; instead, it represents the signed area of the cell

$$
\bar{W}_{i, j}[n]=\text { area of } C_{i, j}^{+}[n]-\text { area of } C_{i, j}^{-}[n]
$$

where $C_{i, j}^{ \pm}[n] \triangleq\{(\beta, \alpha) \quad \in \quad \operatorname{cell}(i, j) \quad$ : the output of $\hat{\gamma}_{\beta, \alpha}$ at time $n$ is \pm 1$\}$. Each component $\bar{\nu}_{i, j}^{*}$ of $\nu^{*}$ now represents the true density value on the cell $(i, j)$. Similarly, $\hat{\nu}[n]$ is now the vector of density values estimated at time $n$. Define $V[n] \triangleq W[n]-W[n-1]$. Define $\hat{y}[n], z[n]$, and $\hat{z}[n]$ as in (4), (6), and (7), respectively. Based on these definitions, the output-based algorithm (5) and the difference-based algorithm (8) can be applied without modification to identify the vector of density values $\nu^{*}$.

\section{B. Parameter Convergence and Persistent Excitation}

Define the parameter error $\tilde{\nu}[n] \triangleq \hat{\nu}[n]-\nu^{*}$. Then, for the output-based algorithm (5) (letting $\gamma=1$ without loss of generality)

$$
\tilde{\nu}[n+1]=F[n] \tilde{\nu}[n]
$$

where $F[n]=\rrbracket_{K}-\left(W[n] W[n]^{T}\right) /\left(W[n]^{T} W[n]\right)$, and $\mathbb{\square}_{K}$ represents the identity matrix of dimension $K$. It is well-known [28] that the convergence of the algorithm (5) depends on persistent excitation (PE) of the sequence $W[n]$. The sequence $W[n]$ is persistently exciting if, there exist an integer $N>0$ and $c_{1}^{\prime}>0, c_{2}^{\prime}>0$, such that for any $n_{0}$

$$
c_{1}^{\prime} \rrbracket_{K} \leq \sum_{n=n_{0}}^{n_{0}+N-1} \frac{W[n] W[n]^{T}}{W[n]^{T} W[n]} \leq c_{2}^{\prime} \rrbracket_{K} .
$$

Due to the preservation of uniform complete observability under output injection [28], [29], from (10), there exist $c_{1}>0, c_{2}>0$ such that for any $n_{0}$

$$
c_{1} \rrbracket_{K} \leq G_{N}\left(n_{0}\right) \leq c_{2} \rrbracket_{K}
$$

where $G_{N}\left(n_{0}\right)$ is the observability grammian of (9) defined as

$$
G_{N}\left(n_{0}\right)=\sum_{n=n_{0}}^{n_{0}+N-1} \frac{\Phi\left[n, n_{0}\right]^{T} W[n] W[n]^{T} \Phi\left[n, n_{0}\right]}{W[n]^{T} W[n]}
$$

and $\Phi\left[n, n_{0}\right]$ is the state transition matrix, $\Phi\left[n, n_{0}\right]=$ $\prod_{k=n_{0}}^{n-1} F[k]$. It can be shown [28] that when (11) is satisfied

$$
\|\tilde{\nu}[n+N]\| \leq \sqrt{1-c_{1}}\|\tilde{\nu}[n]\|
$$

from which exponential convergence to $\nu^{*}$ can be concluded. Similarly, one can write down the error dynamics equation, the PE condition on $V[n]$, and the convergence rate estimate corresponding to the difference-based scheme (8).

The sequences $V[n]$ and $W[n]$ are almost equivalent in the sense that, for any $N>0,\{V[n]\}_{n=1}^{N}$ can be constructed from $\{W[n]\}_{n=0}^{N}$, and conversely, $\{W[n]\}_{n=1}^{N}$ can be constructed from $W[0]$ and $\{V[n]\}_{n=1}^{N}$. However, there are motivations to introduce the difference-based scheme (8). Again let us first consider identification of weighting masses for ease of discussion. In this case, while $W[n]$ has components \pm 1 , the components of $V[n]$ are \pm 2 or 0 . Often times most components of $V[n]$ are 0 since $V_{k}[n] \neq 0$ only if the $k$-th hysteron changed its state at time $n$. This has two consequences: 1) The PE condition of $V[n]$ is easier to analyze than that of $W[n] ; 2)$ the convergence of the difference-based scheme (assuming that PE is satisfied) is expected to be faster than that of the output-based scheme since $z[n]$ carries more specific information about $\nu^{*}$. 
It is of practical interest to express the PE conditions in terms of the input $u[n]$ to the hysteresis operator. In the analysis that follows, it is assumed that the input does not change more than one level during one sampling time. The assumption is not restrictive considering the rate-independence of the Preisach operator.

Theorem 3.1 (Necessary Condition for PE): If $\{V[n]\}$ is PE, then there exists $N>0$, such that for any $n_{0}$, for any $i \in$ $\{1,2, \ldots, L\}, u[n]$ achieves a local maximum at $u_{i+1}$ or a local minimum at $u_{i}$ during the time period $\left[n_{0}, n_{0}+N-1\right]$.

Proof: The PE condition for the difference-based algorithm is equivalent to that $\{V[n]\}_{n=n_{0}}^{n_{0}+N-1}$ spans $\mathbb{R}^{K}$. Let us call a hysteron active at time $n$ if it changes state at time $n$. Since the input changes at most one level each time, if $u[n]>u[n-1]$, the set of active hysterons must have the form $S_{i, j}^{+} \triangleq\{(i, j),(i, j+$ $1), \ldots,(i, i)\}$ for some $i, j$ with $1 \leq i \leq L$ and $1 \leq j \leq i$ [refer to the labeling scheme in Fig. 3(a)], and the components of $V[n]$ corresponding to elements of $S_{i, j}^{+}$are 2 and other components equal 0 . Similarly, if $u[n]<u[n-1]$, the set of active hysterons has the form $S_{i, j}^{-} \triangleq\{(j, j),(j+1, j), \ldots,(i, j)\}$ for some $i, j$, and the components of $V[n]$ corresponding to elements of $S_{i, j}^{-}$ are -2 and other components equal 0 .

If, for some $i^{\prime}, u_{i^{\prime}+1}$ is not a local maximum and $u_{i}^{\prime}$ is not a local minimum, $S_{i^{\prime}, i^{\prime}}^{+}$or $S_{i^{\prime}, i^{\prime}}^{-}$will not become the set of active hysterons during $\left[n_{0}, n_{0}+N-1\right]$. In particular, when the hysteron $\left(i^{\prime}, i^{\prime}\right)$ changes state from -1 to 1 , so does the hysteron $\left(i^{\prime}, i^{\prime}-1\right)$; and when the hysteron $\left(i^{\prime}, i^{\prime}\right)$ changes state from 1 to -1 , so does the hysteron $\left(i^{\prime}+1, i^{\prime}\right)$. This implies that the contribution to the output from the hysteron $\left(i^{\prime}, i^{\prime}\right)$ cannot be isolated and, hence, $\left\{V_{n}\right\}_{n=n_{0}+1}^{n_{0}+N-1}$ does not span $\mathbb{R}^{K}$.

Remark 3.1: From Theorem 3.1, for a Preisach operator with discrete weighting masses, it is necessary that the input $u[n]$ has certain number of reversals for parameter convergence. This is in analogy to (but remarkably different from) the result for linear systems, where the input is required to have at least $n$ frequency components for identification of $n$ parameters [28], [29].

Theorem 3.1 implies that the input levels $u_{1}$ and $u_{L+1}$ must be visited for PE to hold. When the input hits $u_{1}$, all hysterons have output -1 and the Preisach operator is in negative saturation; similarly, when the input hits $u_{L+1}$, the Preisach operator is in positive saturation. For either case all the previous memory is "erased" and the operator is "reset." Starting from these reset points, one can keep track of the memory curve $\psi[n]$ (the state of the Preisach operator) according to the input $u[\cdot]$. Consider an input sequence $\{u[n]\}_{n=n_{a}}^{n_{b}}, n_{a}<n_{b}$. If there exist $n_{1}$, $n_{2}, n_{3}$, and $n_{4}$ with $n_{a} \leq n_{1}<n_{2} \leq n_{3}<n_{4} \leq n_{b}$ such that the memory curve $\psi\left[n_{1}\right]=\psi\left[n_{3}\right]$ and $\psi\left[n_{2}\right]=\psi\left[n_{4}\right]$, we can obtain another input sequence $\left\{u^{\prime}[n]\right\}_{n=n_{a}}^{n_{b}}$ by swapping the section $\{u[n]\}_{n=n_{1}}^{n_{2}}$ with the section $\{u[n]\}_{n=n_{3}}^{n_{4}}$. We write $\{u[n]\}_{n=n_{a}}^{n_{b}} \stackrel{P E}{\equiv}\left\{u^{\prime}[n]\right\}_{n=n_{a}}^{n_{b}}$ (called equivalent in terms of $P E$ ) since the two sequences carry same excitation information for the purpose of parameter identification. The set of all input sequences obtained from $\{u[n]\}_{n=n_{a}}^{n_{b}}$ as explained above (with possibly zero or more than one swappings) form the PE equivalence class of $\{u[n]\}_{n=n_{a}}^{n_{b}}$, denoted as $\{\underline{u[n]}\}_{n=n_{a}}^{n_{b}}$. Note that in particular, $\{u[n]\}_{n=n_{a}}^{n_{b}} \in\{u[n]\}_{n=n_{a}}^{n_{b}}$. We are now ready to present a sufficient condition for PE in terms of the input $u[n]$.
Theorem 3.2 (Sufficient Condition for PE): If there exists $N>0$, such that for any $n_{0}$, one can find $\left\{u^{\prime}[n]\right\}_{n=n_{0}}^{n_{0}+N-1} \in$ $\{u[n]\}_{n=n_{0}}^{n_{0}+N-1}$ satisfying the following: There exist time indices $n_{0} \leq n_{a} \leq n_{1}^{-}<n_{1}^{+}<n_{2}^{-}<n_{2}^{+}<\cdots<n_{i}^{-}<$ $n_{i}^{+}<\cdots \leq n_{b} \leq n_{0}+N-1$ or $n_{0} \leq n_{a} \leq n_{1}^{+}<n_{1}^{-}<$ $n_{2}^{+}<n_{2}^{-}<\cdots<n_{i}^{+}<n_{i}^{-}<\cdots \leq n_{b} \leq n_{0}+N-1$, such that $u^{\prime}\left[n_{i}^{+}\right]$is a local maximum and $u^{\prime}\left[n_{i}^{-}\right]$is a local minimum of $\left\{u^{\prime}[n]\right\}_{n=n_{a}}^{n_{b}}$ for each $i$, these local maxima and minima include all input levels $u_{i}, 1 \leq i \leq L+1$, and either

a) $\quad\left\{u^{\prime}\left[n_{i}^{+}\right]\right\}$is nonincreasing, $u^{\prime}\left[n_{i}^{+}\right] \geq u^{\prime}[n]$ for $n_{i}^{+}<$ $n \leq n_{b}, u^{\prime}\left[n_{i}^{+}\right]$differs from $u^{\prime}\left[n_{i+1}^{+}\right]$by no more than $\Delta_{u}$, and $\left\{u^{\prime}\left[n_{i}^{-}\right]\right\}$is nondecreasing, $u^{\prime}\left[n_{i}^{-}\right] \leq u^{\prime}[n]$ for $n_{i}^{-}<n \leq n_{b}, u^{\prime}\left[n_{i}^{-}\right]$differs from $u^{\prime}\left[n_{i+1}^{-}\right]$by no more than $\Delta_{u}$; or

b) $\quad\left\{u^{\prime}\left[n_{i}^{+}\right]\right\}$is nondecreasing, $u^{\prime}\left[n_{i}^{+}\right] \geq u^{\prime}[n]$ for $n_{i}^{+}>$ $n \geq n_{a}, u^{\prime}\left[n_{i}^{+}\right]$differs from $u^{\prime}\left[n_{i+1}^{+}\right]$by no more than $\Delta_{u}$, and $\left\{u^{\prime}\left[n_{i}^{-}\right]\right\}$is nonincreasing, $u^{\prime}\left[n_{i}^{-}\right] \leq u^{\prime}[n]$ for $n_{i}^{-}>n \geq n_{b}, u^{\prime}\left[n_{i}^{-}\right]$differs from $u^{\prime}\left[n_{i+1}^{-}\right]$by no more than $\Delta_{u}$

then $V[n]$ corresponding to $u[n]$ is PE.

Proof: Construct a new input sequence $\{\bar{u}[n]\}_{n=1}^{\bar{n}}$ (for some $\bar{n}>1)$ which achieves the local maxima $\left\{u^{\prime}\left[n_{i}^{+}\right]\right\}$and the local minima $\left\{u^{\prime}\left[n_{i}^{-}\right]\right\}$with the same order as in $u^{\prime}[n]$, but $\bar{u}[n]$ varies monotonically from a maximum to the next minimum or from a minimum to the next maximum. For such an input, it can be seen through memory curve analysis on the Preisach plane that the corresponding $\{\bar{V}[n]\}_{n=1}^{\bar{n}}$ spans $\mathbb{R}^{K}$. From the way $\bar{u}[n]$ is constructed and the conditions given in the theorem, any vector in $\{\bar{V}[n]\}_{n=1}^{\bar{n}}$ must also be present in $\left\{V^{\prime}[n]\right\}_{n=n_{0}}^{n_{0}+N-1}$ corresponding to $u^{\prime}[n]$. Hence, $\left\{V^{\prime}[n]\right\}$ is PE. Finally, PE of $\{V[n]\}$ follows since $\left\{u^{\prime}[n]\right\}_{n=n_{0}}^{n_{0}+N-1}$ belongs to the PE equivalence class of $\{u[n]\}_{n=n_{0}}^{n_{0}+N-1}$.

Theorem 3.2 is not conservative, and it covers a wide class of PE inputs. For example, it can be easily verified that a (periodic) first order reversal input (see Fig. 4(a) for case $L=4$ ), which has been widely used for identification of Preisach density function [11], and a (periodic) oscillating input with decreasing amplitude (Fig. 4(b) for case $L=4$ ) both satisfy the conditions in Theorem 3.2, and are thus PE. In these two cases, $u[n]$ itself satisfies the conditions imposed for $u^{\prime}[n]$ in the theorem. Fig. 5 shows an example where one can conclude the PE of a periodic $u[n]$ by inspecting a PE equivalent input $u^{\prime}[n]$. Note that Theorem 3.2 does not require $u[n]$ to be periodic, although periodic examples are chosen here for easy illustration.

The PE conditions (Theorems 3.1 and 3.2) can be extended with some modifications to the case of identifying a piecewise uniform density function. In the latter case, the input $u[n]$ may take any value in $\left[u_{1}, u_{L+1}\right]$ and is not restricted to the finite set $\left\{u_{i}\right\}_{i=1}^{L+1}$. Another difference is that the triangular shape of diagonal cells now helps in isolating contributions from these cells (see the simulation results in Fig. 12 and the explanation in Section IV-B).

To be specific, one can show that a necessary condition for PE is that: There exists $\epsilon>0$ and an integer $N>0$, such that for each $i=1, \ldots, L$, for any $n_{0}$, one can find $n$ between $n_{0}$ and $n_{0}+N-1$, with $u[n] \in\left[u_{i}+\epsilon, u_{i+1}-\epsilon\right]$; furthermore, for $L>3$, there are at least $L-1$ input reversals in the block 


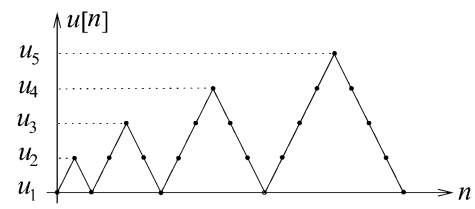

(a)

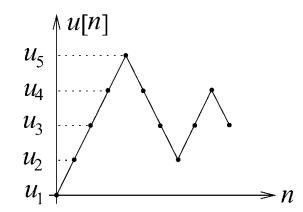

(b)
Fig. 4. Examples of PE inputs ( $L=4$, showing one period). (a) First-order reversal input. (b) Oscillating input with decaying amplitude.
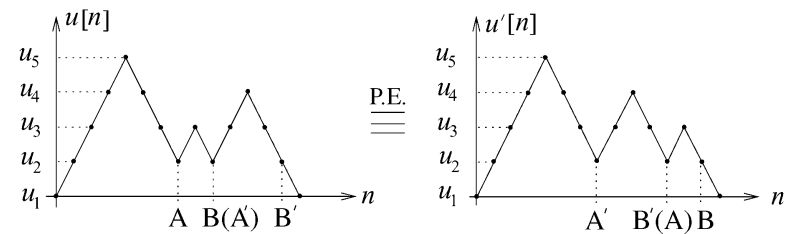

Fig. 5. Example of PE input ( $L=4$, showing one period). The input $u^{\prime}[n]$, PE equivalent to $u[n]$, is obtained by swapping two sections $A-B$ and $A^{\prime}-B^{\prime}$ of $u[n]$.

$\{u[n]\}_{n=n_{0}}^{n_{0}+N-1}$ with each local minimum $\in\left[u_{i}, u_{i+1}-\epsilon\right]$ and each local maximum $\in\left[u_{j}+\epsilon, u_{j+1}\right]$ for some $i, j$. The proof will be analogous to that of Theorem 3.1 except that one should focus on isolation of contributions from inner cells instead of diagonal ones.

To adapt the sufficient condition Theorem 3.2, one changes "include all input levels " to "visit all input bands," "no more than $\Delta_{u}$ " to "within neighboring input bands," and requires each local minimum $\in\left[u_{i}, u_{i+1}-\epsilon\right]$ and each local maximum $\epsilon$ $\left[u_{j}+\epsilon, u_{j+1}\right]$ for some $i, j$. Here by input bands, we mean input ranges $\left[u_{i}, u_{i+1}\right]$ for $i=1, \ldots, L$. The proof will be nearly identical to that of Theorem 3.2.

\section{Comparison of the Output-Based Scheme and the Difference-Based Scheme}

In this section, the output-based scheme is compared with the difference-based one through simulation, where the goal is to identify the Preisach weighting masses. As shown in (12), the minimum eigenvalue of the observability grammian [i.e., $c_{1}$ in (11)] is directly related to the convergence rate of the outputbased scheme. The same statement holds for the differencebased scheme provided that $W[n]$ is replaced with $V[n]$ in the related equations. In Table I, we list the corresponding $\sqrt{1-c_{1}}$ (the bound on parameter error drop over one period) under the two gradient schemes (with $\gamma=1$ ) for different discretization levels $L$ with the (periodic) first order reversal input. From Table I, the difference-based scheme converges faster as expected. Note that although for each $L$, the difference in $\sqrt{1-c_{1}}$ between the two methods appear to be small, it can have significant impact since the error drops as $\left(\sqrt{1-c_{1}}\right)^{n}$ with the period number $n$. Simulation has been conducted for the case $L=10$. Fig. 6(a) compares the decrease of parameter error over periods for the two algorithms when there is no measurement noise, and the conclusion is consistent with Table I.

Despite the apparent advantage of faster convergence, the difference-based scheme is more sensitive to the measurement noise: The noise gets magnified when one takes the output difference (analogous to taking the derivative of a noisy continuous-time signal), and the disturbance is shared only among the

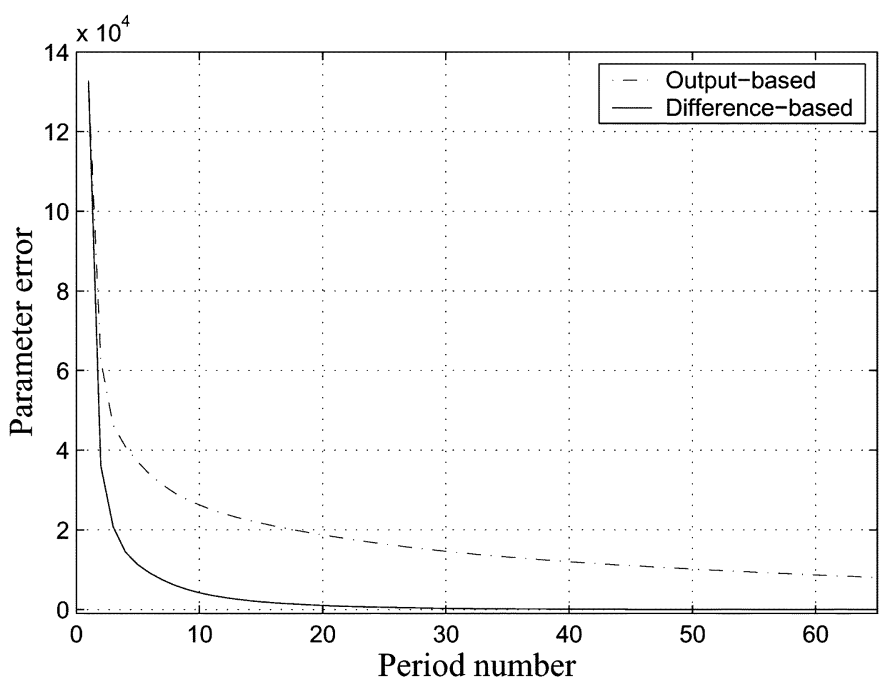

(a)

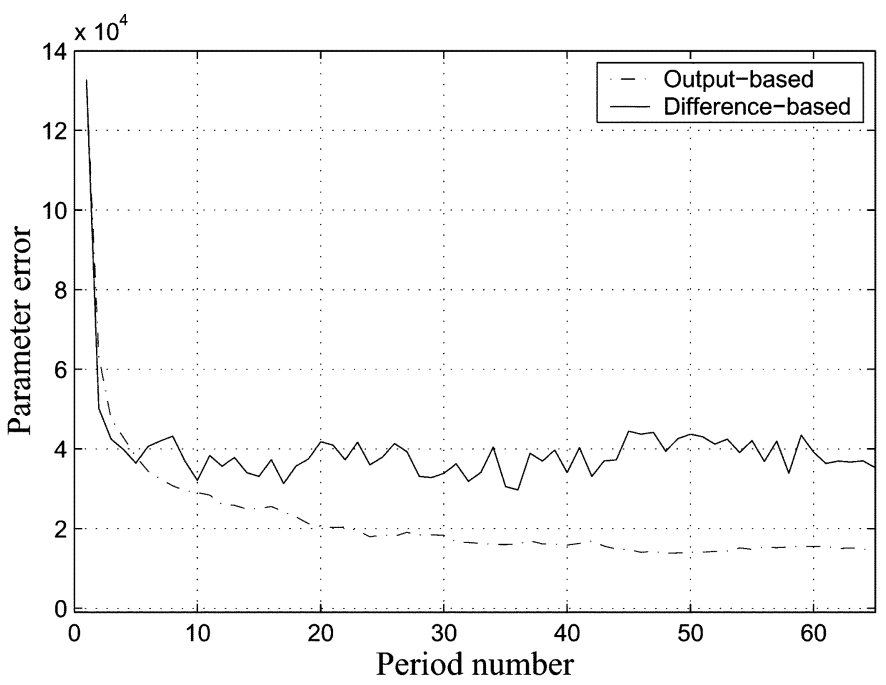

(b)

Fig. 6. Comparison of parameter convergence for the output based algorithm and the difference-based algorithm. (a) Case I: Noiseless measurement. (b) Case II: Noisy measurement.

TABLE I

COMPARISON OF CONVERGENCE RATES FOR THE OUTPUT-BASED ALGORITHM AND THE DIFFERENCE-BASED ALGORITHM

\begin{tabular}{c|c|c}
\hline$L$ & $\begin{array}{c}\sqrt{1-c_{1}} \\
\text { (Output-based) }\end{array}$ & $\begin{array}{c}\sqrt{1-c_{1}} \\
\text { (Difference-based) }\end{array}$ \\
\hline 5 & 0.9631 & 0.9399 \\
\hline 10 & 0.9908 & 0.9784 \\
\hline 15 & 0.9958 & 0.9874 \\
\hline 20 & 0.9976 & 0.9912 \\
\hline 25 & 0.9985 & 0.9933 \\
\hline
\end{tabular}

active hysterons. Simulation in Fig. 6(a) is reconducted where a noise is added to the output, the noise magnitude being $4 \%$ of the saturation output of the Preisach operator. From Fig. 6(b), in this case, the parameter error will not converge to zero under either of the two algorithms. However, the ultimate identification error of the output-based algorithm is much lower than that of the difference-based scheme. For this reason, it is recommended that the output-based algorithm be used when the measurement noise is not negligible. 


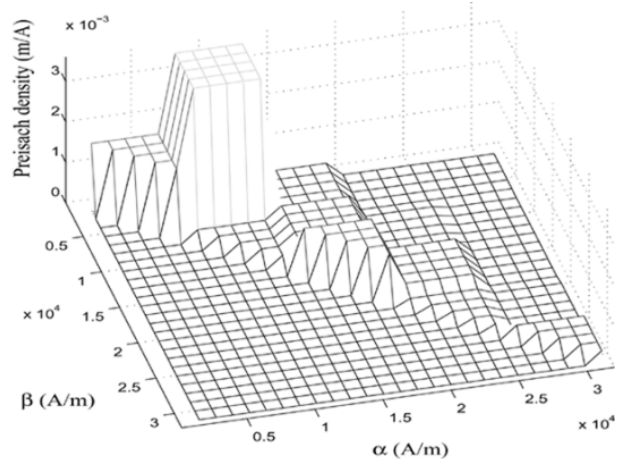

(a)

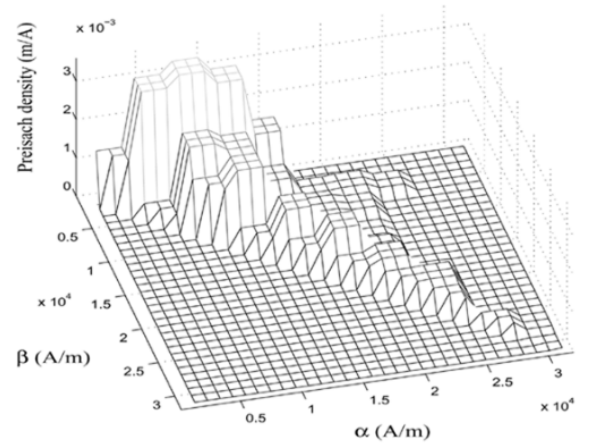

(b)

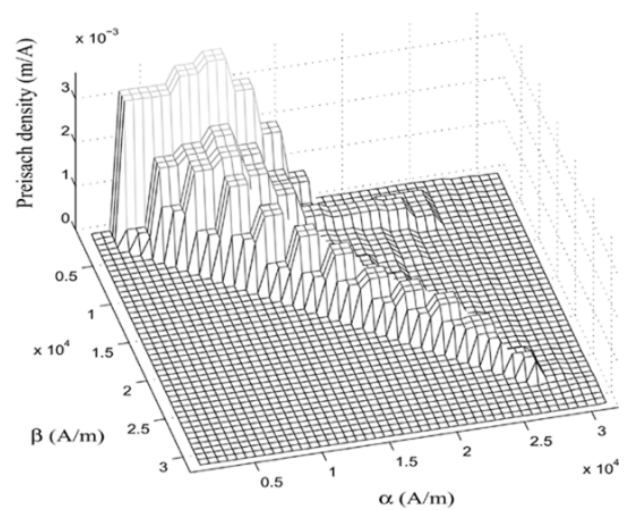

(c)

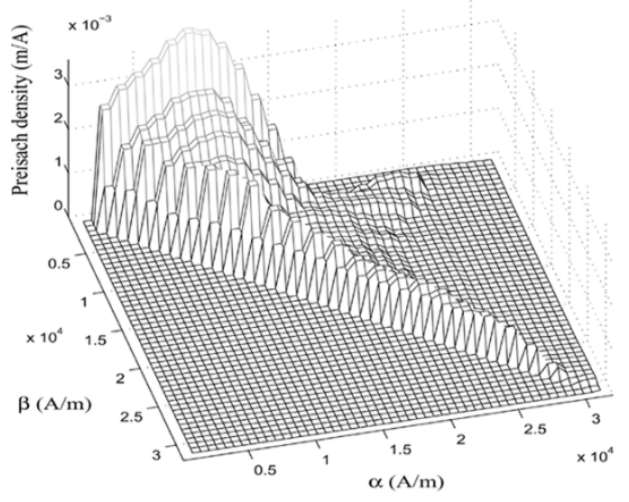

(d)

Fig. 7. Preisach density function identified for different levels of discretization $L$. (a) $L=5$. (b) $L=10$. (c) $L=15$. (d) $L=25$.

\section{Experimental Results Based on a Magnetostrictive Actuator}

Experiments have been conducted on a magnetostrictive (Terfenol-D) actuator to examine the identification schemes. Refer to [20] for a description of the experimental setup. The displacement output $y$ of the actuator is controlled by the magnetic field

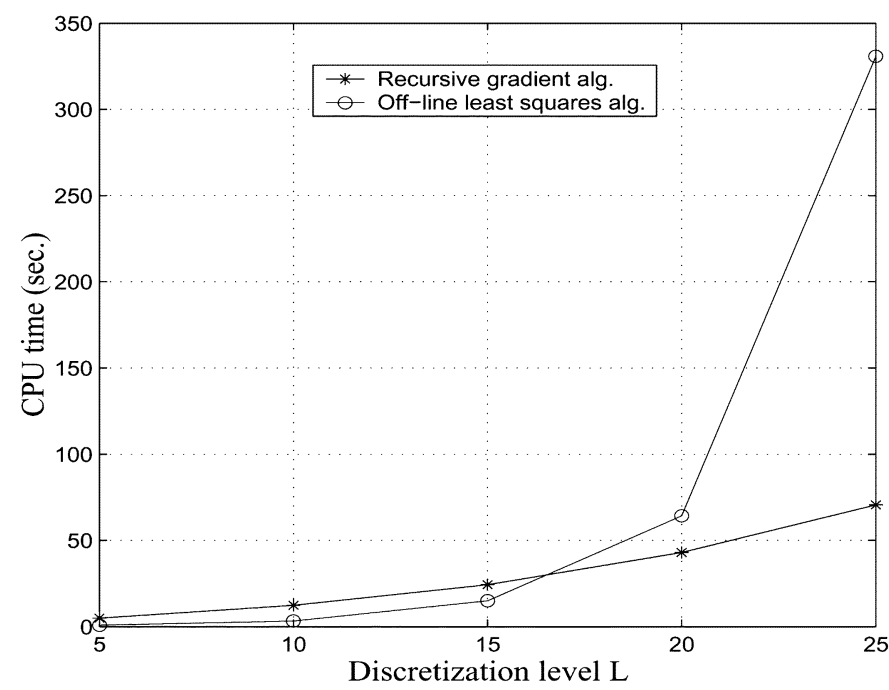

Fig. 8. CPU time used in the recursive gradient algorithm and the offline least-squares algorithm.

generated through the current $I$ in a coil. When operated in a low frequency range (typically below $5 \mathrm{~Hz}$ ), the displacement $y$ can be related to the bulk magnetization $M$ by a square law $y=a_{1} M^{2}$ for some constant $a_{1}>0$, and the current $I$ can be related to the magnetic field $H$ along the rod direction by $H=c_{0} I$, where $c_{0}$ is the coil factor [30]. Then, the magnetostrictive hysteresis between $y$ and $I$ is fully captured by the ferromagnetic hysteresis between $M$ and $H$, which is modeled by the Preisach operator.

In the experiment a periodic first-order reversal input (with sufficiently dense distribution of turning points) is applied. The choice of the discretization level $L$ is of practical importance. Fig. 7 shows the identified density distribution for different discretization levels after eight periods. The output-based gradient algorithm is used with $\gamma=1$.

Although it is expected that the higher discretization level $L$, the higher model accuracy, there are two factors supporting a moderate value of $L$ in practice: The computational complexity and the sensor accuracy level. Since the number of cells on a discretization grid scales as $L^{2}$, so is the computational complexity of the recursive identification algorithm. It should also be noted that, from Table I, the convergence rate $\sqrt{1-c_{1}}$ decreases as $L$ increases. Fig. 8 shows the CPU time used in recursive identification for different discretization levels. To obtain the CPU time, the recursive algorithm is carried out again using the collected data (the current $I$ and the displacement $D$ ) of eight periods on a Dell laptop Inspiron 4150. Also, shown in Fig. 8 is the CPU time it takes to compute the Preisach density function using an offline, constrained least squares algorithm [8], where the data of one period were used. From Fig. 8, the square law for the recursive algorithm is evident. The offline algorithm becomes prohibitively time-consuming as $L$ gets large, due to the increasing complexity of solving a constrained optimization problem of many variables.

In the presence of the sensor noise and unmodeled dynamics, higher discretization level may not necessarily lead to improved performance. Fig. 9 compares the measured hysteresis loops against the predicted loops based on the identified parameters for different $L$. Although the scheme with $L=10$ achieves 


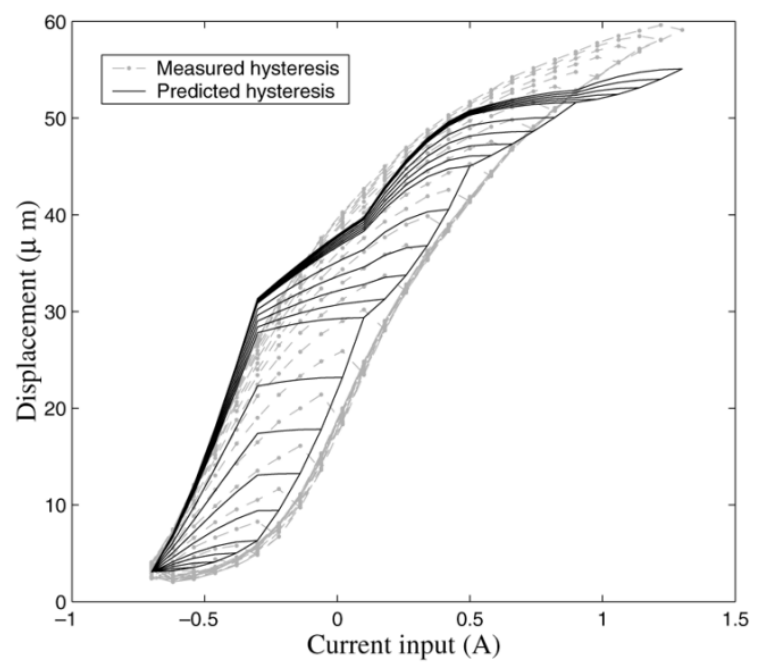

(a)

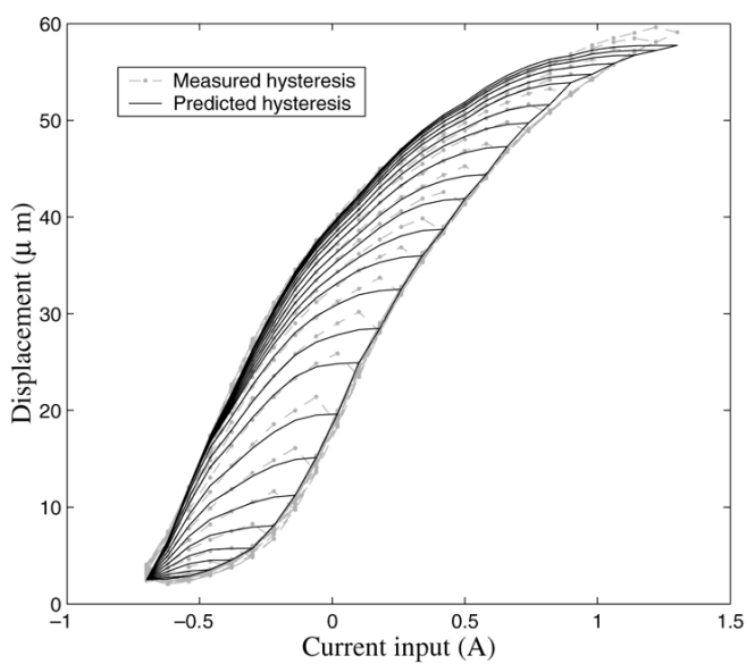

(b)

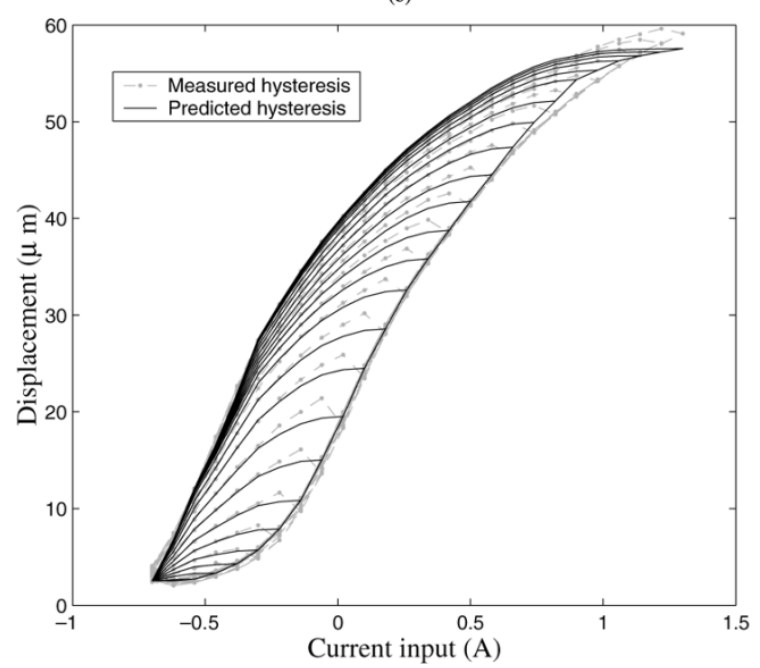

(c)

Fig. 9. Comparison of measured hysteresis loops with predicted loops based on the identified Preisach density function. (a) $L=5$. (b) $L=10$. (c) $L=15$.

much better match than the scheme with $L=5$, there is little improvement when $L$ is increased to 15 . Hence for the Terfenol-D actuator (and the LVDT sensor used), it is determined that $L=10$ is an appropriate discretization level for the Preisach operator.

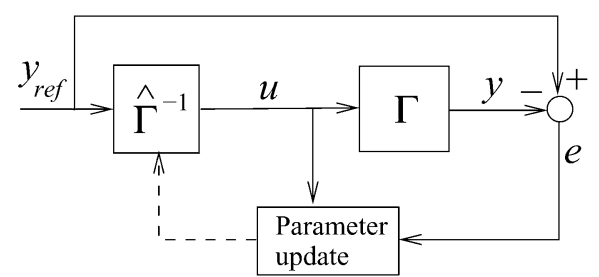

Fig. 10. Schematic of adaptive inverse control.

\section{ADAPTIVE INVERSE CONTROL}

\section{A. Adaptive Inverse Control Scheme}

Fig. 10 shows a schematic of adaptive inverse control, where $\Gamma$ represents a Preisach operator with a piecewise uniform density function, and $\hat{\Gamma}$ represents the Preisach operator with the current density estimate. The input to $\Gamma$ is obtained through inversion of $\hat{\Gamma}$. The error between the reference trajectory $y_{\text {ref }}[n]$ and the achieved trajectory $y[n]$ is then used to update the parameter estimate $\hat{\nu}[n]$ and thus the inverse model $\hat{\Gamma}^{-1}$, where the output-based identification scheme (5) is used. Later on we shall also discuss the performance of adaptive inverse compensation using the difference-based parameter update (8), which typically fails to achieve asymptotic tracking.

The inversion scheme for $\hat{\Gamma}$ is now briefly discussed. Let $\psi[n-1]$ be the memory curve of $\hat{\Gamma}$ (and of $\Gamma$ ) at time $n-1$, and $\hat{y}_{\text {sat }}[n]$ be the saturation output corresponding to $\hat{\nu}[n]$. The input $u[n]$ to $\Gamma$ is generated through the following inversion algorithm.

A. If $y_{\text {ref }}[n]<-\hat{y}_{\text {sat }}[n], u[n]=u_{\min }$;

B. If $y_{\text {ref }}[n]>\hat{y}_{\text {sat }}[n], u[n]=u_{\max }$;

C. Otherwise $u[n]=\hat{\Gamma}^{-1}\left[y_{\text {ref }}[n], \psi[n-1]\right]$, where $\hat{\Gamma}^{-1}$ is the (right) inverse of $\hat{\Gamma}$ constructed as in [20].

Let $\hat{y}[n]$ be the predicted output of $\hat{\Gamma}$. When $\left|y_{\text {ref }}[n]\right|>$ $\hat{y}_{\text {sat }}[n]$, there exists no $u[n]$ such that $\hat{\Gamma}[u[n], \psi[n-1]]=$ $y_{\text {ref }}[n]$. Item $\mathrm{A}$ (item $\mathrm{B}$, respectively) makes sure that $\hat{y}[n]$ equals $-\hat{y}_{\text {sat }}[n]$ ( $\hat{y}_{\text {sat }}[n]$, respectively) and, hence, is closest to $y_{\text {ref }}[n]$. On the other hand, when $\left|y_{\text {ref }}[n]\right| \leq \hat{y}_{\text {sat }}[n], y_{\text {ref }}[n]$ can be inverted exactly [20] and, therefore, $\hat{y}[n]=y_{\text {ref }}[n]$.

Theorem 4.1: Assume that the true Preisach operator has a piecewise uniform density function. Denote by $\nu^{*}$ the $K$-dimensional vector of true densities, and by $y_{\text {sat }}$ the saturation output corresponding to $\nu^{*}$. Let the output-based gradient algorithm be used for the parameter update. Then, the following hold.

1) For any reference trajectory $y_{\text {ref }}[\cdot]$ with $y_{\text {ref }}[n] \in$ $\left[-y_{\text {sat }}, y_{\text {sat }}\right]$, the parameter estimate $\hat{\nu}[n] \rightarrow \hat{\nu}_{\infty}$ for some $\hat{\nu}_{\infty}$, and the tracking error $e[n] \triangleq$ $y[n]-y_{\text {ref }}[n] \rightarrow 0$ as $n \rightarrow \infty$.

2) Assume that the density $\bar{\nu}_{1,1}$ of the cell $(1,1)$ is positive. Let $y_{\text {ref }}[\cdot]$ be periodic of period $N$ that visits $-y_{\text {sat }}$, and without loss of generality $y_{\text {ref }}[1]=-y_{\text {sat }}$. Define $u_{r}[n] \triangleq \Gamma^{-1}\left[y_{\text {ref }}[\cdot], \psi_{0}\right][n]$ with $\psi_{0}$ the memory curve corresponding to the negative saturation, where $\Gamma^{-1}$ is as constructed in [20]. Then, $u_{r}[\cdot]$ is also periodic with period $N$, and $u_{r}[1]=u_{\min }$. Let the vector of signed areas of cells corresponding to $u_{r}[\cdot]$ be $W_{r}[\cdot]$ (which is also periodic), and the null 
space of $\left[W_{r}[1] \cdots W_{r}[N]\right]^{T}$ be $\mathcal{N}_{r}$. Then, the parameter estimate $\hat{\nu}[n] \rightarrow \mathcal{N}_{r}+\nu^{*} \triangleq\left\{x+\nu^{*}: x \in \mathcal{N}_{r}\right\}$. In particular, if $\left\{W_{r}[n]\right\}_{n=1}^{N}$ spans $\mathbb{R}^{K}, \hat{\nu}[n] \rightarrow \nu^{*}$. Analogous results hold if $\bar{\nu}_{L, L}>0$ and $y_{\text {ref }}[\cdot]$ visits $y_{\text {sat }}$.

\section{Proof:}

1) $\quad$ Define $\tilde{\nu}[n] \triangleq \hat{\nu}[n]-\nu^{*}$, and $\delta[n] \triangleq \tilde{\nu}[n]^{T} \tilde{\nu}[n]$. From the output-based gradient algorithm (letting $\gamma=1$ without loss of generality)

$$
\begin{aligned}
\delta[n+1] & =\delta[n]-\frac{\tilde{\nu}[n]^{T} W[n] W[n]^{T} \tilde{\nu}[n]}{W[n]^{T} W[n]} \\
& =\delta[n]-\frac{(y[n]-\hat{y}[n])^{2}}{W[n]^{T} W[n]} \leq \delta[n] .
\end{aligned}
$$

Since $\delta[n] \geq 0, \delta[n] \rightarrow \delta_{\infty}$ as $n \rightarrow \infty$. This immediately results in $\hat{\nu}[n] \quad \rightarrow \quad \hat{\nu}_{\infty}$ for some $\hat{\nu}_{\infty}$. Summing (13) over $n$ leads to $\sum_{n=0}^{\infty}(y[n]-\hat{y}[n])^{2} /\left(W[n]^{T} W[n]\right)=\delta[0]-\delta_{\infty}<$ $\infty$, which implies $(y[n]-\hat{y}[n])^{2} /\left(W[n]^{T} W[n]\right) \rightarrow$ 0 . Since $W[n]^{T} W[n]<C$ for some constant $C$,

$$
|y[n]-\hat{y}[n]| \rightarrow 0, \text { as } n \rightarrow \infty .
$$

The Preisach operator $\hat{\Gamma}$ based on $\hat{\nu}[n]$ can be exactly inverted (hence $\hat{y}[n]=y_{\text {ref }}[n]$ ) except for the following two cases: a) $\hat{y}_{\text {sat }}[n]<y_{\text {ref }}[n] \leq y_{\text {sat }}$, and b) $-y_{\text {sat }} \leq y_{\text {ref }}[n]<-\hat{y}_{\text {sat }}[n]$, where $\hat{y}_{\text {sat }}[n]$ denotes the saturation output corresponding to $\hat{\nu}[n]$. For case a), the input under the inversion algorithm is $u[n]=$ $u_{\max }$ with $\hat{y}[n]=\hat{y}_{\text {sat }}[n], y[n]=y_{\text {sat }}$, and hence $\left|y[n]-y_{\text {ref }}[n]\right|<|y[n]-\hat{y}[n]|$. The same conclusion holds for the case $b$ ). It then follows from (14) that the tracking error $e[n]$ approaches 0 as $n \rightarrow \infty$.

2) Since $y_{\text {ref }}[k N+1]=-y_{\text {sat }}$ for each $k$ and $\bar{\nu}_{1,1}>$ $0, u_{r}[k N+1]=u_{\min }$ and the state of the Preisach operator is reset at time $k N+1$. The periodicity of $u_{r}[\cdot]$ then follows from that of $y_{\text {ref }}[\cdot]$ and the inverse algorithm. From the first part of the theorem, $\mid y[n]-$ $y_{\text {ref }}[n] \mid \rightarrow 0$ and hence $y[k N+1] \rightarrow-y_{\text {sat }}$ as $k \rightarrow \infty$. Again, from $\bar{\nu}_{1,1}>0$, the input $u[k N+1]$ approaches $u_{\min }$ as $k \rightarrow \infty$, and $u[k N+m] \rightarrow u_{r}[m]$ for $1 \leq$ $m \leq N$. As a consequence, $W[k N+m] \rightarrow W_{r}[m]$. Since as $k \rightarrow \infty$

$$
W[k N+m]^{T} \tilde{\nu}[k N+m] \rightarrow 0, \quad \text { for } 1 \leq m \leq N
$$

we conclude $W_{r}[m]^{T} \hat{\nu}_{\infty}=W_{r}[m]^{T} \nu^{*}, 1 \leq m \leq$ $N$, i.e., $\hat{\nu}_{\infty} \in \mathcal{N}_{r}+\nu^{*}$. When $\left\{W_{r}[n]\right\}_{n=1}^{N}$ spans $\mathbb{R}^{K}, \mathcal{N}_{r}=\{0\}$ and hence $\hat{\nu}[n] \rightarrow \nu^{*}$. Analogous arguments can be used for the case where $\bar{\nu}_{L, L}>0$ and $y_{\text {ref }}[\cdot]$ visits $y_{\text {sat }}$.

Remark 4.1: Note that for the Preisach operator $\Gamma$ to have a unique (right) inverse, it is in general required that all diagonal cells have strictly positive density values [13]. However, when a particular inversion algorithm (e.g., the one in [20]) is used, the inverse trajectory can be made unique even if some diagonal density values are zero. Therefore, $u_{r}[\cdot]$ in the statement of Theorem 4.1 is well defined.
What happens if the difference-based algorithm (8) is used for parameter update in Fig. 10? Following the similar arguments as in the proof of Theorem 4.1, one can show $\hat{\nu}[n] \rightarrow \hat{\nu}_{\infty}$ for some $\hat{\nu}_{\infty}$, and $|z[n]-\hat{z}[n]| \rightarrow 0$, where $z[n]$ and $\hat{z}[n]$ are as defined in (6) and (7), respectively. This implies

$$
\left|\left(y[n]-\hat{y}\left[n^{-}\right]\right)-(y[n-1]-\hat{y}[n-1])\right| \rightarrow 0 .
$$

From $\hat{\nu}[n] \rightarrow \hat{\nu}_{\infty}, \hat{y}\left[n^{-}\right] \rightarrow \hat{y}[n]$. Even with a strong assumption $\left|y_{\text {ref }}[n]\right| \leq \hat{y}_{\text {sat }}[n]$ as $n \rightarrow \infty$, one can only conclude $y[n]-y_{\text {ref }}[n] \rightarrow C_{e}$ for some constant $C_{e}$. Therefore, one cannot expect to achieve asymptotic tracking if the difference-based scheme is adopted.

\section{B. Simulation and Experimental Results}

Simulation and experiments have been conducted to illustrate Theorem 4.1. Fig. 11(a) shows the simulation results of tracking a sinusoidal signal with amplitude $y_{\text {sat }}$ using the output-based adaptive inverse scheme. One can see that the tracking error goes to zero. Fig. 11(b) shows the simulation results of tracking where density parameters experience random changes of up to $20 \%$ of their original values at $t=5 \mathrm{~s}$, and the adaptive scheme is seen to suppress the tracking error quickly.

The simulation in Fig. 11(a) is continued for 160 periods, and Fig. 12 compares the parameter estimates with the true parameter values at the end of simulation. Note that the individual density values do not converge [Fig. 12(a)]. For this particular reference trajectory, the asymptotic input $u_{r}[\cdot]$ will be periodic varying between $u_{\min }$ and $u_{\max }$ without other reversals. Hence, when $u_{r}$ is increasing, for each fixed $i$, the components of $W_{r}$ corresponding to cells $(i, 1), \ldots,(i, i-1)$ are equal; when $u_{r}$ is decreasing, for each fixed $j$, the components of $W_{r}$ corresponding to cells $(j+1, j), \ldots,(L, j)$ are equal. What separates a diagonal cell from other cells of the row (or the column) is its triangular shape. As a result, one expect that the densities of diagonal cells will be correctly identified, and the sum of densities of cells in each row (or column) excluding the diagonal element will also be correctly identified. This is verified in Fig. 12(b), where by "aggregate cell density values," we mean the quantities $\bar{\nu}_{i, i}^{*}, \sum_{j^{\prime}=1}^{i-1} \bar{\nu}_{i, j^{\prime}}^{*}$, and $\sum_{i^{\prime}=j+1}^{L} \bar{\nu}_{i^{\prime}, j}^{*}$ with $1 \leq i, j \leq L$.

To further verify Part 2 of Theorem 4.1, simulation is also conducted for tracking an oscillating signal with decaying amplitude for 128 periods (Fig. 13). The corresponding input to the desired signal is PE, and indeed Fig. 14 shows the convergence of individual cell density values.

Experimental results for tracking a sinusoidal signal are shown in Fig. 15 with two different adaptation constants $\gamma$. In these experiments and other experiments reported hereafter, the discretization level $L=10$. The current input applied to the magnetostrictive actuator is also displayed in addition to the reference trajectory, the achieved trajectory, and the tracking error. It can be seen that when $\gamma$ is smaller, the trajectory converges to the steady state slower but with smaller tracking error due to lower sensitivity to the noise. Fig. 16 plots the achieved displacement versus the desired one at the steady-state (after 10 s) for $\gamma=0.2$. The plot would overlap well with the $45^{\circ}$ line except for a small segment in the region $[55,60] \mu \mathrm{m}$, where the error is about $\pm 0.5 \mu \mathrm{m}$. Considering the sensor precision, almost perfect tracking is achieved for the full operational 

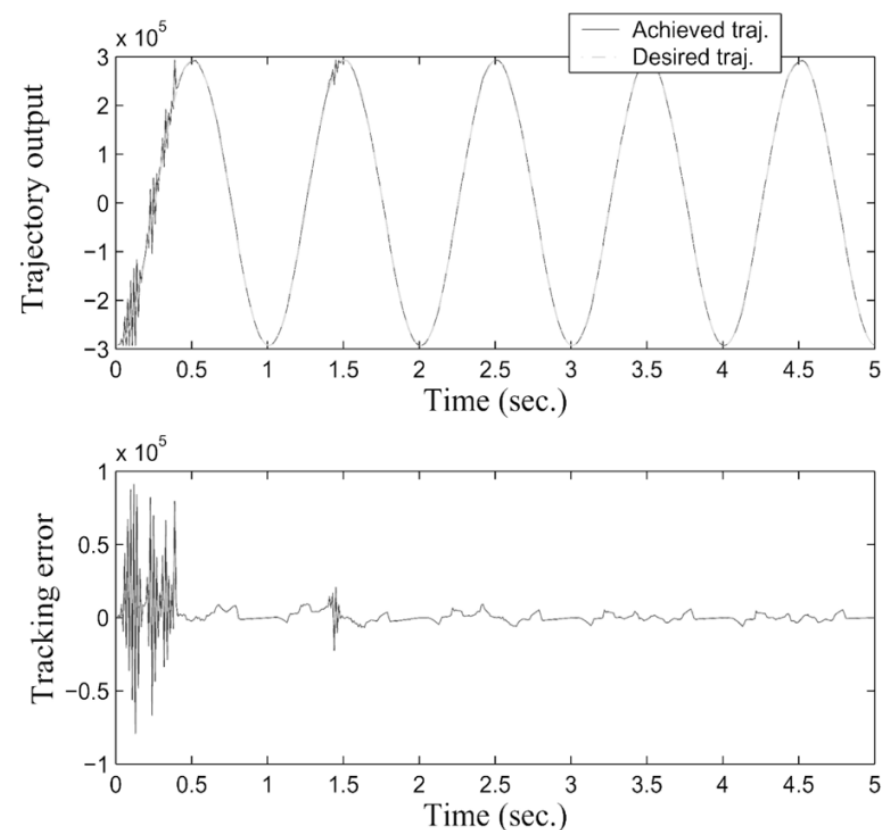

(a)
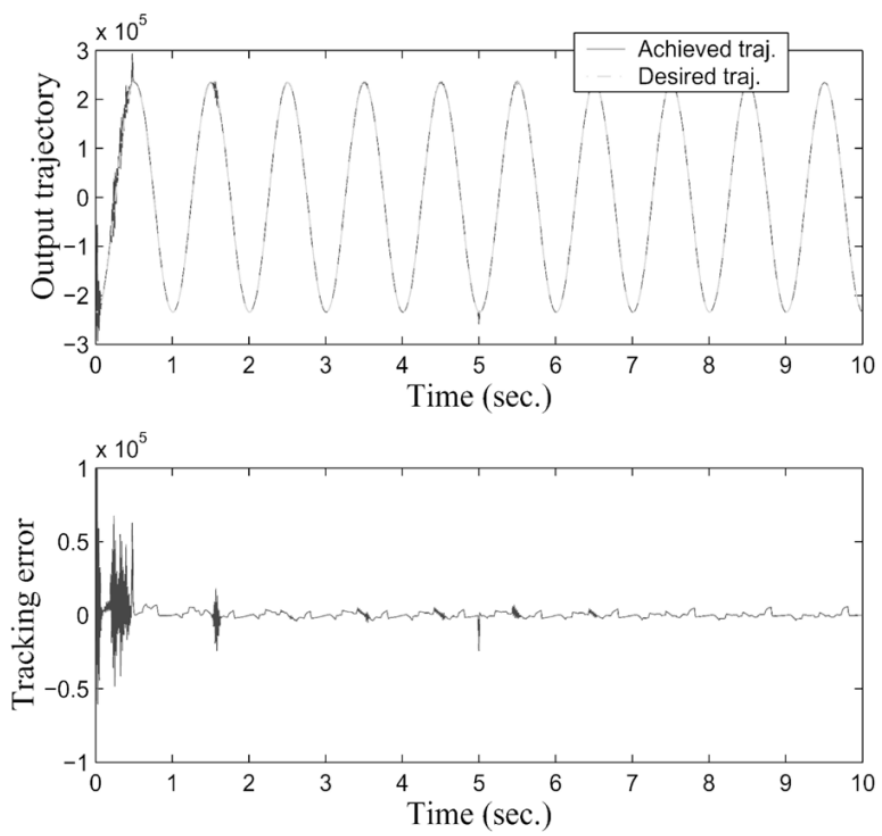

(b)

Fig. 11. (a) Simulation results of tracking a sinusoidal signal with amplitude $y_{\text {sat }}(L=10)$. (b) Simulation results of tracking a sinusoidal signal with sudden parameter change at $t=5 \mathrm{~s}$. $(L=10)$.

range $(60 \mu \mathrm{m})$ of the actuator. Note that $\pm 0.5 \mu \mathrm{m}$ error was also achieved by Natale et al. [10], however, there the tracking range was $25 \mu \mathrm{m}$-about half of the range reported here.

If the reference trajectory does not cover $\pm y_{\text {sat }}$, Theorem 4.1 says that the tracking error still goes to zero, but one cannot say more about the parameter convergence. In this case, there is no reset mechanisms during the adaptation, and depending on the initial conditions of the system and the adaptation process, the final steady-state input trajectories can be different (while the output trajectories are all consistent with the reference trajectory). Essentially, there may exist multiple minor loops that satisfy the output requirement. To confirm this, two experiments

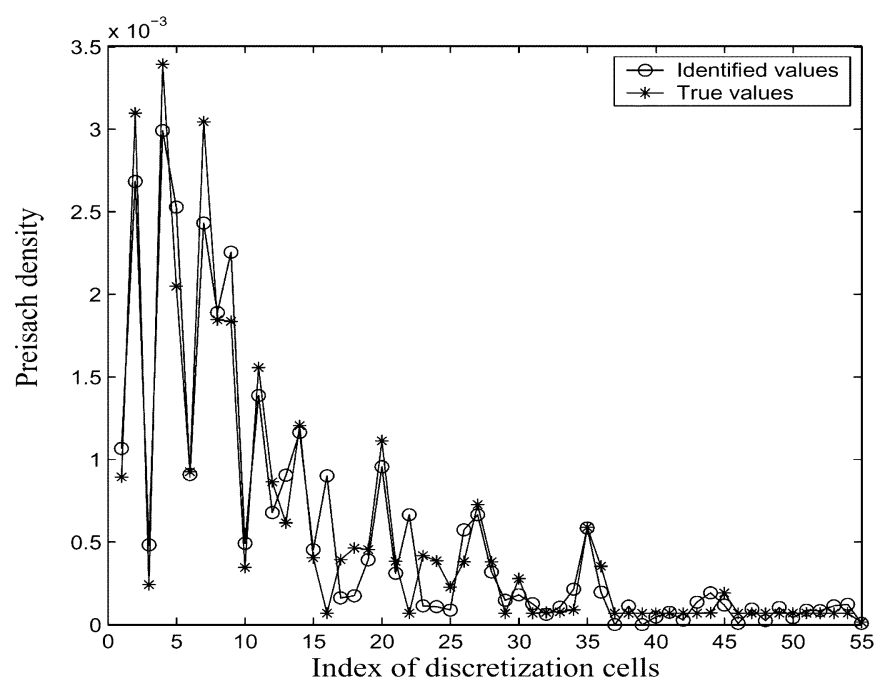

(a)

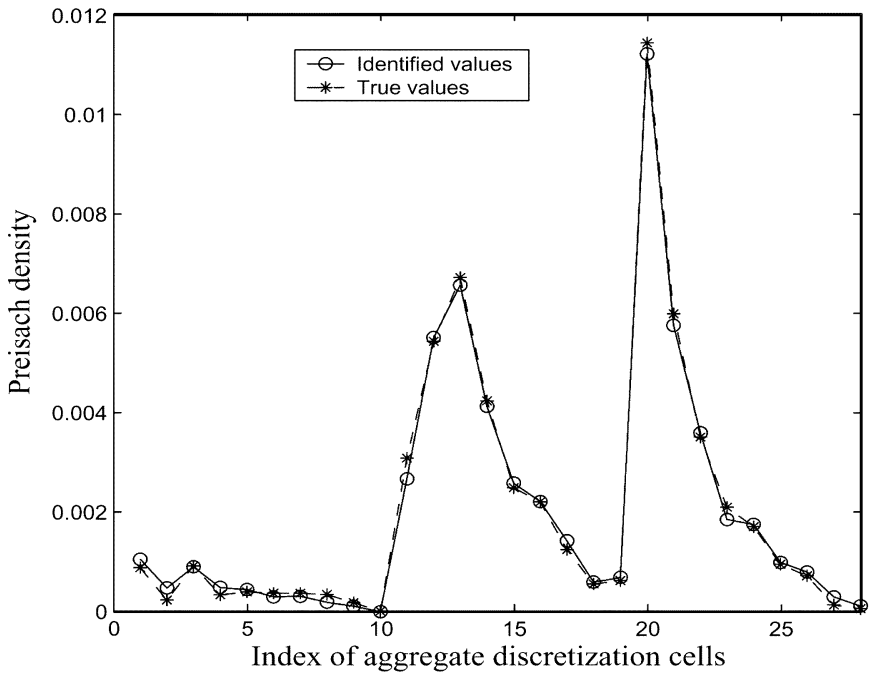

(b)

Fig. 12. Comparison of identified parameter values with true values after tracking a sinusoidal signal for 160 periods $(L=10)$ for (a) individual cell density values and (b) aggregate cell density values.

are conducted to track a sinusoidal trajectory of amplitude 15 $\mu \mathrm{m}$ (and a dc offset of $30 \mu \mathrm{m}$ ), one starting from the negative saturation and the other from the positive saturation. The steady-state current inputs obtained through adaptive inverse control are different, yet they are both able to track the desired trajectory. Fig. 17 plots the achieved displacement versus the current for the two cases, and one can see that the ranges of current input differ by about $5 \%$, but the displacement ranges are consistent.

Finally, an experiment is performed to verify the analysis at the end of Section IV-A. Here, the difference-based scheme (8) is used to update the density estimate during adaptation. Fig. 18 shows the comparison of the desired trajectory and the achieved one, which clearly indicates that this scheme cannot achieve asymptotic tracking.

\section{CONCLUSION AND DiscusSiONS}

This paper has been focused on recursive identification and adaptive inverse control of hysteresis in smart materials. A 

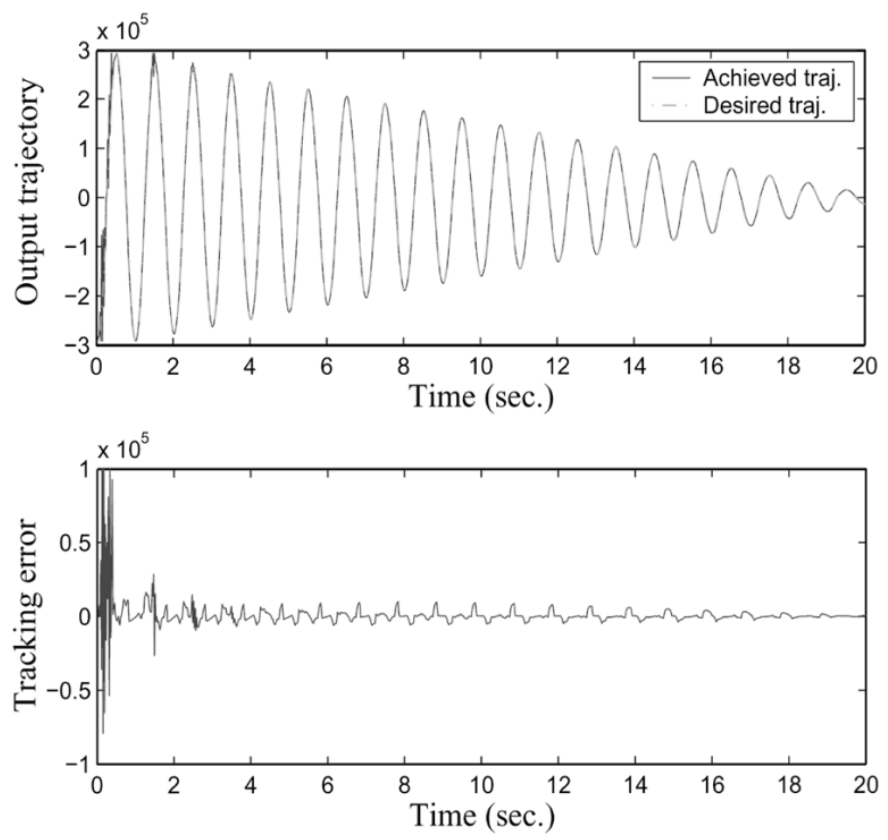

Fig. 13. Simulation results of tracking an oscillating signal with decaying amplitude ( $L=10$, showing the first period).

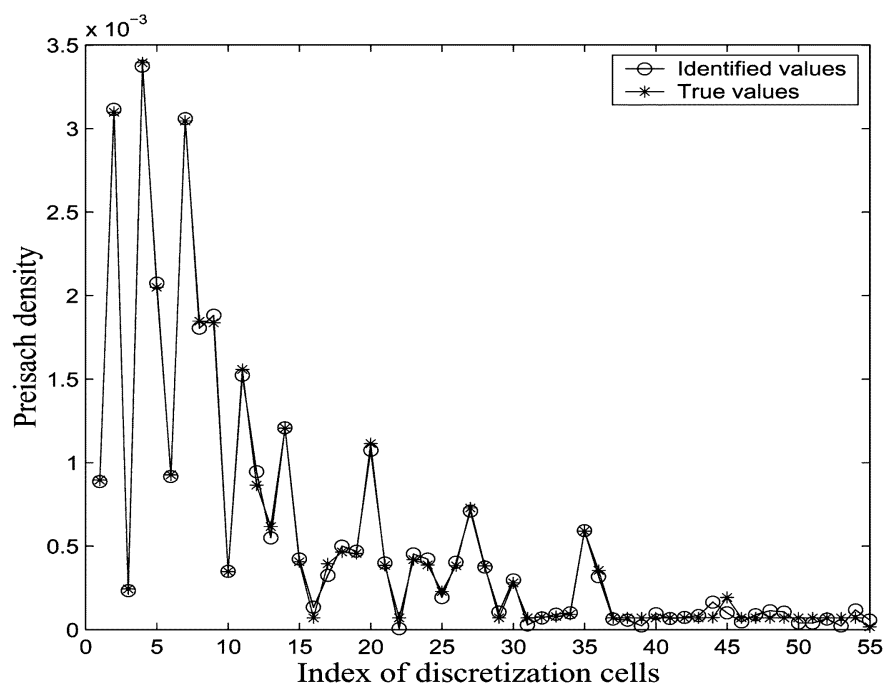

Fig. 14. Comparison of identified density values of individual cells with true values after tracking an oscillating signal with decaying amplitude for 128 periods $(L=10)$.

Preisach operator with piecewise uniform density function was used to approximate smart material hysteresis. To facilitate analysis and presentation, a Preisach operator with discrete weighting masses was also treated. A necessary condition and a sufficient condition for the parameter convergence were presented in terms of the hysteresis input. In contrast to the results for linear systems, the conditions here are centered around the local maxima/minima (hence, reversals) of the input. Asymptotic tracking under the output-based algorithm was established, and the behavior of parameter convergence was discussed for periodic reference trajectories. Although uniform discretization of the Preisach plane was considered, the results are applicable to the case of nonuniform discretization, as one can easily verify. Nonuniform discretization could be
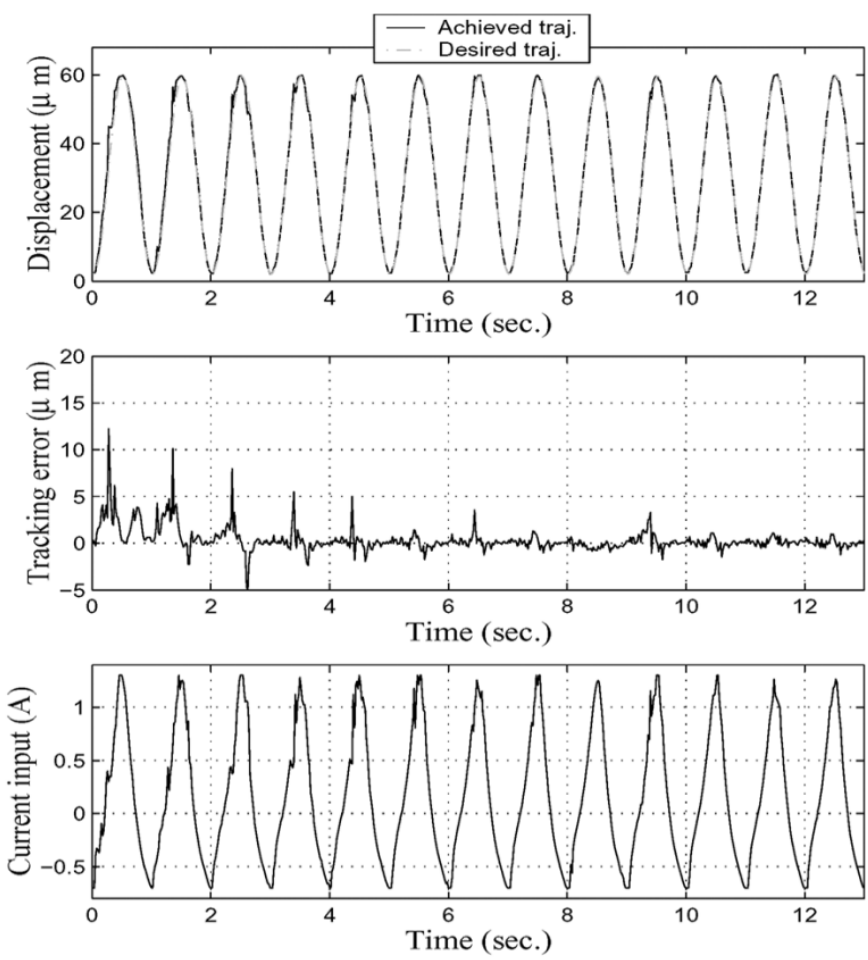

(a)
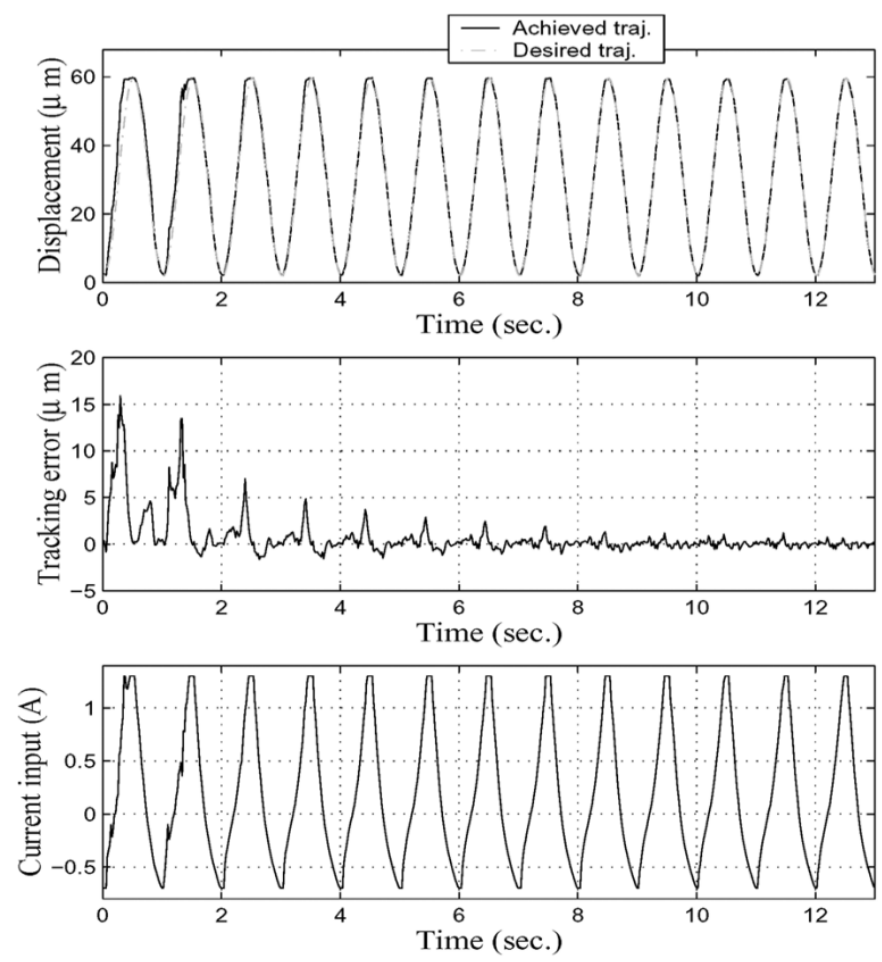

(b)

Fig. 15. Experimental results of tracking a sinusoidal reference trajectory. (a) $\gamma=0.5$. (b) $\gamma=0.2$.

useful when the actual density function varies a lot in certain region while changes little elsewhere.

Two types of adaptive gradient identification algorithms were compared. Although the difference-based scheme cannot be used for adaptive tracking, it can be a viable choice for recursive parameter identification when the measurement noise 


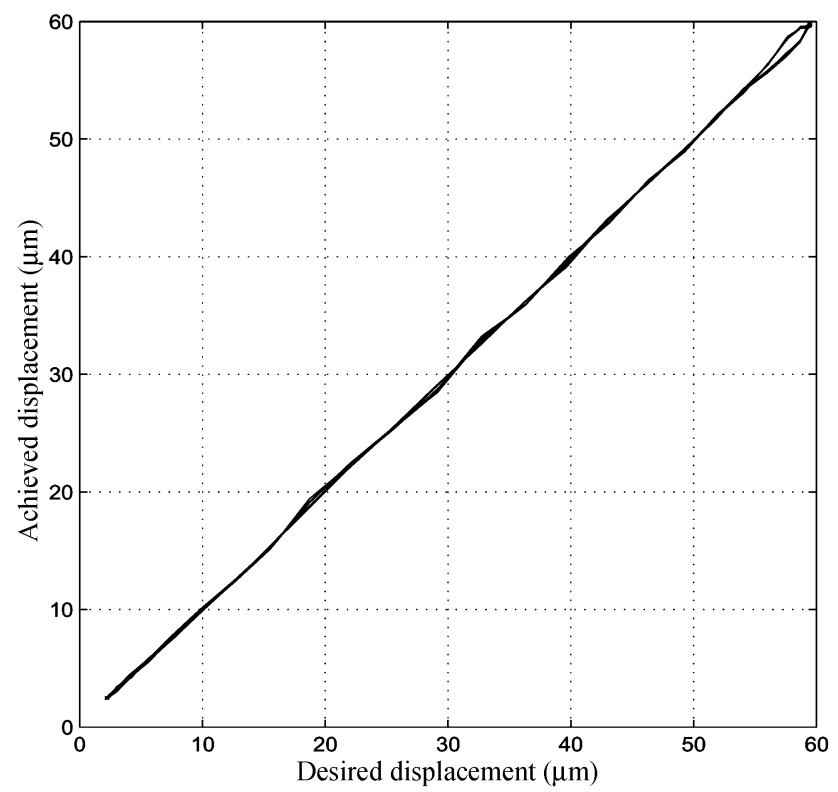

Fig. 16. Achieved displacement versus desired displacement (over the full operational range of the actuator) after $10 \mathrm{sec}$. of adaptation.

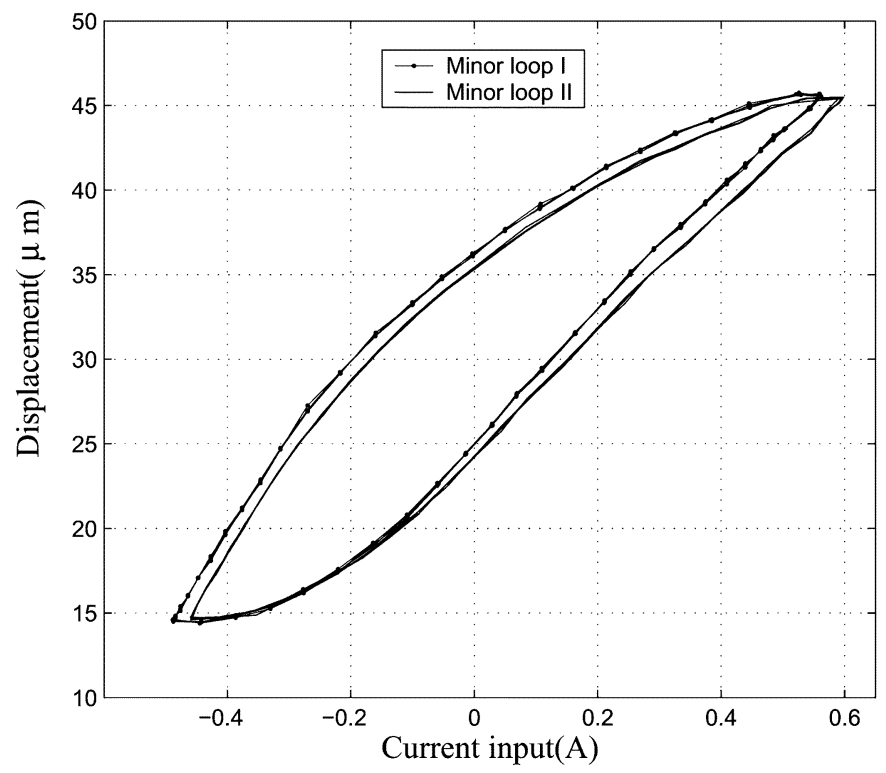

Fig. 17. Two minor loops corresponding to tracking a sinusoidal signal with amplitude less than $y_{\text {sat }}$ (at steady state).

is low. The introduction of a difference-based algorithm also helped in analyzing the PE conditions.

Computational complexity is one of the primary concerns when it comes to real-time implementation. The inversion algorithm used in this paper was specifically developed for Preisach operators with piecewise uniform densities. For each desired output value, the algorithm finds its (exact) inverse in a finite number of iterations and thus is very efficient [20]. The level $L$ of discretization determines the computational cost in both inversion and adaptation. $L$ should be chosen based on available computational resources, the sensor noise level, and the control requirement. In particular, for our experimental setup,

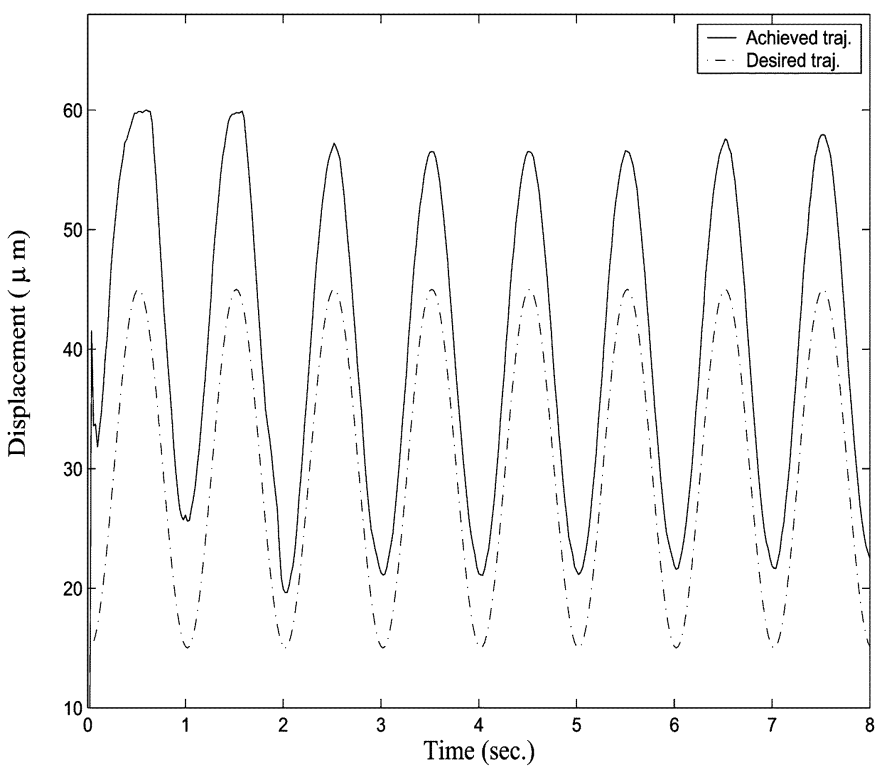

Fig. 18. Experimental result showing that the difference-based adaptive scheme cannot achieve asymptotic tracking.

with $L=10$ the adaptive inversion scheme was able to virtually cancel out the hysteresis effect in the actuator.

The asymptotic tracking result was based on the assumption of time-invariant models. However, simulation indicated that the proposed scheme could adapt quickly should model parameters change. It is expected that the scheme will work well in practice as long as the hysteresis behavior changes at a relatively slow time scale.

For future work, it will be of interest to extend the results reported here to the cases where the hysteresis output is not directly measurable. Such cases happen if, e.g., the high-frequency dynamics of the smart material actuator is not negligible and hence a rate-independent hysteresis operator alone is inadequate to capture the dynamic and hysteretic behavior [20], [31], or the actuator is used to control some other plant.

\section{ACKNOWLEDGMENT}

The authors wish to thank the anonymous reviewers for their constructive comments that helped in improving the paper.

\section{REFERENCES}

[1] S. O. R. Moheimani and G. C. Goodwin, "Guest editorial introduction to the special issue on dynamics and control of smart structures," IEEE Trans. Control Syst. Technol., vol. 9, pp. 3-4, 2001.

[2] D. C. Jiles and D. L. Atherton, "Theory of ferromagnetic hysteresis," $J$. Magnet. Magn. Mater, vol. 61, pp. 48-60, 1986.

[3] A. A. Adly, I. D. Mayergoyz, and A. Bergqvist, "Preisach modeling of magnetostrictive hysteresis," J. Appl. Phys., vol. 69, no. 8, pp. 5777-5779, 1991.

[4] D. Hughes and J. T. Wen, "Preisach modeling and compensation for smart material hysteresis," in Active Materials and Smart Structures, ser. SPIE, G. L. Anderson and D. C. Lagoudas, Eds., 1994, vol. 2427, pp. $50-64$.

[5] J. Schäfer and H. Janocha, "Compensation of hysteresis in solid-state actuators," Sensors Actuators A, vol. 49, no. 1-2, pp. 97-102, 1995.

[6] P. Ge and M. Jouaneh, "Tracking control of a piezoceramic actuator," IEEE Trans. Control Syst. Technol., vol. 4, pp. 209-216, 1996.

[7] R. B. Gorbet, D. W. L. Wang, and K. A. Morris, "Preisach model identification of a two-wire SMA actuator," in Proc. IEEE Int. Conf. Robotics and Automation, 1998, pp. 2161-2167. 
[8] X. Tan, R. Venkataraman, and P. S. Krishnaprasad, "Control of hysteresis: Theory and experimental results," in Modeling, Signal Processing, and Control in Smart Structures, ser. SPIE, V. S. Rao, Ed., 2001, vol. 4326, pp. 101-112.

[9] D. Croft, G. Shed, and S. Devasia, "Creep, hysteresis, and vibration compensation for piezoactuators: Atomic force microscopy application," $J$. Dyna. Syst., Measure., Control, vol. 123, no. 1, pp. 35-43, 2001.

[10] C. Natale, F. Velardi, and C. Visone, "Identification and compensation of Preisach hysteresis models for magnetostrictive actuators," Physica $B$, vol. 306, pp. 161-165, 2001.

[11] I. D. Mayergoyz, Mathematical Models of Hysteresis. New York: Springer-Verlag, 1991.

[12] M. A. Krasnosel'skii and A. V. Pokrovskii, Systems With Hysteresis. New York: Springer-Verlag, 1989.

[13] A. Visintin, Differential Models of Hysteresis. New York: SpringerVerlag, 1994

[14] H. T. Banks, A. J. Kurdila, and G. Webb, "Identification of hysteretic control influence operators representing smart actuators, part I: Formulation," Math. Prob. Eng., vol. 3, no. 4, pp. 287-328, 1997.

[15] W. S. Galinaitis and R. C. Rogers, "Control of a hysteretic actuator using inverse hysteresis compensation," in Mathematics and Control in Smart Structures, ser. SPIE, V. Varadan, Ed., 1998, vol. 3323, pp. 267-277.

[16] K. Kuhnen and H. Janocha, "Adaptive inverse control of piezoelectric actuators with hysteresis operators," in Proc. European Control Conf. (ECC), Karsruhe, Germany, 1999, paper F 0291.

[17] G. Tao and P. V. Kokotović, "Adaptive control of plants with unknown hysteresis," IEEE Trans. Autom. Control, vol. 40, pp. 200-212, 1995.

[18] R. C. Smith, "Inverse compensation for hysteresis in magnetostrictive transducers," CRSC, North Carolina State Univ., Raleigh, NC, Tech. Rep. CRSC-TR98-36, 1998.

[19] L. Sun, C. Ru, W. Rong, L. Chen, and M. Kong, "Tracking control of piezoelectric actuator based on a new mathematical model," J. Micromech. Microeng., vol. 14, pp. 1439-1444, 2004.

[20] X. Tan and J. S. Baras, "Modeling and control of hysteresis in magnetostrictive actuators," Automatica, vol. 40, no. 9, pp. 1469-1480, 2004.

[21] G. V. Webb, D. C. Lagoudas, and A. J. Kurdila, "Hysteresis modeling of SMA actuators for control applications," J. Intell. Mater. Syst. Struct., vol. 9, no. 6, pp. 432-448, 1998 .

[22] K.-H. Hoffmann, J. Sprekels, and A. Visintin, "Identification of hysteretic loops," J. Comput. Phys., vol. 78, pp. 215-230, 1988.

[23] R. V. Iyer and M. E. Shirley, "Hysteresis parameter identification with limited experimental data," IEEE Trans. Magn., vol. 40, pp. 3227-3239, 2004

[24] X. Tan and J. S. Baras, "Recursive identification of hysteresis in smart materials," in Proc. Amer. Control Conf., Boston, MA, 2004, pp. 3857-3862.

[25] — - "Adaptive inverse control of hysteresis in smart materials," in Proc. IFAC Symp. Nonlinear Control Systems, Stuttgart, Germany, 2004

[26] M. Brokate and J. Sprekels, Hysteresis and Phase Transitions. New York: Springer-Verlag, 1996

[27] X. Tan, "Control of smart actuators," Ph.D. dissertation, Univ. Maryland, College Park, MD, 2002.

[28] G. C. Goodwin and K. S. Sin, Adaptive Filtering, Prediction and Control. Upper Saddle River, NJ: Prentice-Hall, 1984.

[29] S. Sastry and M. Bodson, Adaptive Control: Stability, Convergence, and Robustness. Upper Saddle River, NJ: Prentice-Hall, 1989.

[30] R. Venkataraman, "Modeling and adaptive control of magnetostrictive actuators," Ph.D. dissertation, Univ. Maryland, College Park, MD, 1999.

[31] D. Davino, C. Natale, S. Pirozzi, and C. Visone, "Phenomenological dynamic model of a magnetostrictive actuator," Physica B, vol. 343, pp. 112-116, 2004.

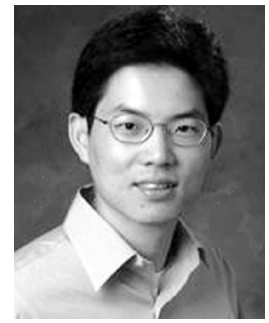

Xiaobo Tan (S'97-M'02) was born in Danyang, China, in 1972. He received the B.S. and M.S. degrees in automatic control from Tsinghua University, Beijing, China, in 1995 and 1998, respectively, and the Ph.D. degree in electrical and computer engineering from the University of Maryland, College Park, in 2002. His Ph.D. dissertation was focused on modeling and control of hysteresis in smart actuators.

From September 2002 to July 2004, he was a Research Associate with the Institute for Systems Research (ISR), the University of Maryland. In August 2004, he joined the Department of Electrical and Computer Engineering, Michigan State University, East Lansing, as an Assistant Professor. His research interests include modeling and control of smart materials and micro-electromechanical systems, distributed control of networked systems, and numerical integration of dynamical systems on manifolds.

Dr. Tan was an ISR Systems Fellow from 1998 to 2002. He was a finalist for the Best Student Paper Award at the 2002 IEEE Conference on Decision and Control, and a co-recipient of the Best Poster Award at the Greater WashingtonBaltimore Area MEMS Alliance Special Topics Symposium in April 2003.

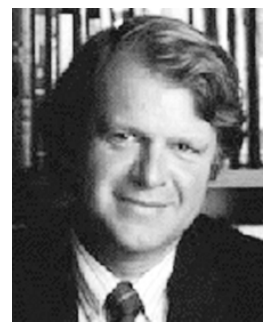

John S. Baras (S'73-M'73-SM'83-F'84) received the B.S. degree in electrical engineering from the National Technical University of Athens, Athens, Greece, in 1970, and the M.S. and Ph.D. degrees in applied mathematics from Harvard University, Cambridge, MA, in 1971 and 1973, respectively.

Since 1973, he has been with the Electrical and Computer Engineering Department and the Applied Mathematics Faculty, the University of Maryland, College Park, where he is currently a Professor and the Lockheed Martin Chair in Systems Engineering. He was the Founding Director of the Institute for Systems Research (ISR, one of the first six NSF ERCs) from 1985 to 1991, and has been the Director of the Center for Hybrid and Satellite Communication Networks (a NASA Center for the Commercial Development of Space) since 1991. His research interests include integration of logic programming and nonlinear programming for tradeoff analysis, object-oriented modeling, validation, and verification of complex systems models and engineering designs, hybrid, satellite and wireless communication networks, integrated network management systems, network security, stochastic systems, robust control of nonlinear systems, real-time parallel architectures for nonlinear signal processing, intelligent control systems, expert and symbolic systems for control and communication systems synthesis, biomimetic algorithms and systems for signal processing and sensor networks, and intelligent manufacturing of smart materials. He has published over 350 technical articles on control, communication and computing systems, and was the Editor of the book Recent Advances in Stochastic Calculus (New York: Springer-Verlag, 1990).

Dr. Baras has served on the Board of Governors of the IEEE Control Systems Society, IEEE Engineering R\&D Committee, Aerospace Industries Association Advisory Committee on Advanced Sensors, and the IEEE Fellow evaluation committee. He is currently serving on the Editorial Boards of Mathematics of Control, Signals, and Systems, of Systems and Control: Foundations and Applications, the IMA Journal of Mathematical Control and Information, and Systems Automation-Research and Applications. He has received the 1978, 1983, and 1993 Naval Research Laboratory Research (Alan Berman) Publication Awards, the 1980 Outstanding Paper Award of the IEEE Control Systems Society, the 1996 Outstanding Paper Award at Design SuperCon, and the 2002 Best Paper Award at the 23rd Army Science Conference. 